ESAIM: COCV 22 (2016) 148-168

DOI: $10.1051 / \mathrm{cocv} / 2015005$
ESAIM: Control, Optimisation and Calculus of Variations

www.esaim-cocv.org

\title{
A VISCO-ELASTO-PLASTIC EVOLUTION MODEL WITH REGULARIZED FRACTURE
}

\author{
LUKÁŠ JAKABČIN ${ }^{1}$
}

\begin{abstract}
We study a model for visco-elasto-plastic deformation with fracture, in which fracture is approximated via a diffuse interface model. We show that a discretized (in time) quasistatic evolution, converges to a solution of the continuous (in time) evolution, proving existence of a solution to our model.
\end{abstract}

Mathematics Subject Classification. 49J40, 49J45, 74C10, 74R20.

Received June 25, 2014.

Published online October 6, 2015.

\section{INTRODUCTION}

This paper deals with a visco-elasto-plastic model with regularized fracture. The model predicting fracture is based on Griffith's criterion [13] that crack path and crack growth are determined by the competition between the elastic energy and the energy dissipated to produce a crack. The variational approach to fracture mechanics and a mathematical model have been developed by Francfort and Marigo [12], based on this idea. This approach has been then adapted by Dal Maso and Toader [9] to the study of fracture problems in elasto-plastic materials with cracks in the case of planar small strain elasto-plasticity where the fracture is represented by the compact crack set $\Gamma \subset \bar{\Omega}$ verifying an irrevesibility condition.

In Larsen, Ortner, Suli [16] existence and convergence results are proved for a regularized model of dynamic brittle fracture based on the Ambrosio-Tortorelli approximation. This model couples an elastodynamic equation with regularized fracture. Babadjian, Francfort, Mora [3], and Babadjian, Mora [2] study the approximation of dynamic and quasistatic evolution problems in elasto-plasticity via viscosity regularization.

The goal of this paper is to propose a model that takes into consideration three dissipative terms: plastic flow, fracture and viscous dissipation. This is motivated by the modeling of the Earth crust considered as a visco-elasto-plastic solid in which cracks are allowed to propagate. This hypothesis is qualitatevely supported by analogue 2D-experiments of Peltzer and Tapponnier [17] that show faults propagation in a layer of plasticine. We study from mathematical point of view a model in $\mathbb{R}^{2}$ of visco-elasto-plastic material that may account for the behaviour observed in the plasticine experiments, but our results extend to any dimension. The main objective is to understand by which mechanisms energy can be dissipated in such model.

\footnotetext{
Keywords and phrases. Plasticity, regularized fracture, viscous dissipation.

1 Laboratoire Jean Kuntzmann, 51 rue des Mathématiques, Campus de Saint Martin d'Hères, BP 53, 38041 Grenoble cedex 09,

France. lukas.jakabcin@imag.fr
} 
In this model the fracture is obtained via Ambrosio-Tortorelli regularization. We only consider fracture via a diffuse interface model. In other words, the geometry of possible cracks is captured by a function $v$ with values between 0 and $1, v=1$ in the healthy parts that do not contain cracks. The length of the cracks, a quantity that contributes to the total energy, is approximated via a functional introduced by Ambrosio and Tortorelli [1]. In other words, the continuous model is obtained coupling visco-elasto-plastic behaviour with regularized fracture evolution of the model of Larsen, Ortner and Suli [16].

A convenience of a such model is the fact, that it can be studied numerically thanks to the presence of regularized fracture. For more details, see [6-8] for the numerical studies in elastic case and [4,15] for the numerical studies of our models in the case of traction and plasticine experiments.

In this paper, we propose the mathematical analysis of a such model via a semi-discrete time procedure. We approximate a continuous time evolution, via semi-discrete time evolutions obtained solving incremental minimum problems. We then prove an existence result to the continuous model when a time discretization parameter converges to zero. The main difficulty is pass to the limit in the discrete plastic flow rule and discrete crack propagation condition. For this reason, we prove particularly a strong compactness result for elastic strain (Prop. 3.12). As in [2], we prove that the discrete-time elastic strain $e_{h}^{+}$converges strongly in $L^{2}\left(0, T_{f}, L^{2}\left(\Omega, \mathbb{M}_{\text {sym }}^{2 \times 2}\right)\right)$, but the presence of $v$ in our model requires the analysis and control of some additional terms associated to $v$.

The paper is organised as follows. After a short introduction, Section 1 is devoted to the definitions, mathematical and mechanical settings necessary to the description of our model. The main result is then presented in Section 3. Firstly, we prove the existence of solutions for discrete minimum problem in Proposition 3.2 and Proposition 3.4. Then, we study the convergence of these approximate evolutions as the time step $h \rightarrow 0$. The main result of this paper is presented as follows: There exists at least one limit evolution $(u, v, e, p)$ that satisfies initial and irreversibility conditions, the equilibrium equation, plastic flow rule and crack propagation condition (Thm. 3.1).

\section{Formulation OF THE MODEL}

\subsection{Preliminaries and mathematical setting}

Throughout the paper, $\Omega$ is a bounded connected open set in $\mathbb{R}^{2}$ with Lipschitz boundary $\partial \Omega=\partial \Omega_{D} \cup \partial \Omega_{N}$ where $\partial \Omega_{D}, \partial \Omega_{N}$ are disjoint open sets in $\partial \Omega$ and $\mathcal{H}^{1}\left(\partial \Omega_{D}\right)>0$. Given $T_{f}>0$, we denote by $L^{p}\left(\left(0, T_{f}\right), X\right)$, $W^{k, p}\left(\left(0, T_{f}\right), X\right)$, the Lebesgue and Sobolev spaces involving time (see [11], p. 285), where $\mathrm{X}$ is a Banach space. We will usually write $u(t):=u(., t)$ for $u \in W^{k, p}\left(\left(0, T_{f}\right), X\right)$.

The set of symmetric $2 \times 2$ matrices is denoted by $\mathbb{M}_{\mathrm{sym}}^{2 \times 2}$. For $\xi, \zeta \in \mathbb{M}_{\mathrm{sym}}^{2 \times 2}$ we define the scalar product between matrices $\zeta: \xi:=\sum_{i j} \zeta_{i j} \xi_{i j}$, and the associated matrix norm by $|\xi|:=\sqrt{\xi: \xi}$. Let A be the fourth order tensor of Lamé coefficients. We assume that for some constants $0<\alpha_{1} \leq \alpha_{2}<\infty$, they satisfy the ellipticity conditions

$$
\forall e \in \mathbb{M}_{\mathrm{sym}}^{2 \times 2}, \quad \alpha_{1}|e|^{2} \leq A e: e \leq \alpha_{2}|e|^{2} .
$$

For $e \in \mathbb{M}_{\text {sym }}^{2 \times 2}$ and $x \in \Omega$, we define $|e|_{A(x)}^{2}:=A(x) e: e$ and $\|e\|_{A}^{2}:=\int_{\Omega}|e|_{A}^{2} \mathrm{~d} x$.

We recall that the mechanical unknowns of our model are the displacement field $u: \Omega \times\left[0, T_{f}\right] \rightarrow \mathbb{R}^{2}$, the elastic strain $e: \Omega \times\left[0, T_{f}\right] \rightarrow \mathbb{M}_{\text {sym }}^{2 \times 2}$, the plastic strain $p: \Omega \times\left[0, T_{f}\right] \rightarrow \mathbb{M}_{\text {sym }}^{2 \times 2}$. We assume $u$ and $\nabla u$ remain small. So that the relation bewteen the deformation tensor $E$ and the displacement field is given by

$$
E u:=\frac{1}{2}\left(\nabla u+\nabla u^{T}\right) .
$$

We also assume that $E u$ decomposes as an elastic part and a plastic part

$$
E u=e+p .
$$


We also define the set of kinematically admissible fields by

$$
\begin{aligned}
A_{a d m} & :=\left\{(u, e, p) \in H^{1}\left(\Omega, \mathbb{R}^{2}\right) \times L^{2}\left(\Omega, \mathbb{M}_{\mathrm{sym}}^{2 \times 2}\right) \times L^{2}\left(\Omega, \mathbb{M}_{\mathrm{sym}}^{2 \times 2}\right):\right. \\
E u & \left.=e+p \quad \text { a.e. in } \Omega, u=0 \quad \text { a.e. on } \partial \Omega_{D}\right\} .
\end{aligned}
$$

We denote $H_{D}^{1}:=\left\{u \in H^{1}\left(\Omega, \mathbb{R}^{2}\right) ; u=0\right.$ on $\left.\partial \Omega_{D}\right\}$. For a fixed constant $\tau>0$, we define $K:=\left\{q \in \mathbb{M}_{\text {sym }}^{2 \times 2} ;|q| \leq \tau\right\}$ and $H: \mathbb{M}_{\text {sym }}^{2 \times 2} \rightarrow[0, \infty]$ the support function of $K$ by

$$
H(p):=\sup _{\theta \in K} \theta: p=\tau|p| .
$$

For $\eta>0$, the elastic energy is defined as

$$
\begin{aligned}
\mathcal{E}_{e l}: L^{2}\left(\Omega, \mathbb{M}_{\mathrm{sym}}^{2 \times 2}\right) \times H^{1}(\Omega, \mathbb{R}) \rightarrow \mathbb{R} \\
(e, v) \longmapsto \mathcal{E}_{e l}(e, v)=\frac{1}{2} \int_{\Omega}\left(v^{2}+\eta\right) A e: e \mathrm{~d} x .
\end{aligned}
$$

In the following, we will define an evolution as a limit of time discretizations with a time step $h$. In fact, $p$ and $p_{0}$ represent the plastic deformation at 2 consecutive time steps, so that $\frac{p-p_{0}}{h} \sim \dot{p}$. In the same way, $u$ and $u_{0}$ represent displacement field at 2 consecutive time steps, so that $\frac{u-u_{0}}{h} \sim \dot{u}$. The plastic dissipated energy is defined, by

$$
\begin{aligned}
& \mathcal{E}_{p}: L^{2}\left(\Omega, \mathbb{M}_{\mathrm{sym}}^{2 \times 2}\right) \times L^{2}\left(\Omega, \mathbb{M}_{\mathrm{sym}}^{2 \times 2}\right) \rightarrow \mathbb{R} \\
& \left(p, p_{0}\right) \longmapsto \mathcal{E}_{p}\left(p, p_{0}\right)=\int_{\Omega} H\left(p-p_{0}\right) \mathrm{d} x
\end{aligned}
$$

Given $\beta>0$, the viscoelastic dissipated energy is defined by

$$
\begin{aligned}
& \mathcal{E}_{v e}: H^{1}\left(\Omega, \mathbb{R}^{2}\right) \times H^{1}\left(\Omega, \mathbb{R}^{2}\right) \rightarrow \mathbb{R} \\
& \left(u, u_{0}\right) \longmapsto \mathcal{E}_{v e}\left(u, u_{0}\right)=\frac{\beta}{2 h} \int_{\Omega}\left(E u-E u_{0}\right):\left(E u-E u_{0}\right) \mathrm{d} x .
\end{aligned}
$$

The Griffith surface energy is approximated by the phase-field surface energy

$$
\begin{aligned}
\mathcal{E}_{S}: H^{1}(\Omega, \mathbb{R}) & \rightarrow \mathbb{R} \\
v & \longmapsto \mathcal{E}_{S}(v)=\int_{\Omega} \varepsilon|\nabla v|^{2} \mathrm{~d} x+\int_{\Omega} \frac{(1-v)^{2}}{4 \varepsilon} \mathrm{d} x .
\end{aligned}
$$

It is shown in [5] that in the elastic anti-plane case, where the displacement reduces to a scalar and $E u$ reduces to $\nabla u$, the Ambrosio-Tortorelli functional

$$
\mathcal{E}_{\varepsilon}(\nabla u, v)=\mathcal{E}_{e l}(\nabla u, v)+\mathcal{E}_{S}(v),
$$

$\Gamma$-converges, as $0<\eta \ll \epsilon \rightarrow 0$, to the Griffith energy $\mathcal{G}$, where

$$
\mathcal{G}(u):=\frac{1}{2} \int_{\Omega} A|\nabla u|^{2} \mathrm{~d} x+\mathcal{H}^{1}(S(u)) .
$$

Here, $S(u)$ denotes the discontinuity set of $u$, and $\mathcal{H}^{1}$ is the 1 -dimensional Hausdorff measure. In the case of $n$-dimensional elasticity the $\Gamma$-convergence result for Ambrosio-Tortorelli approximation has been proved recently by Iurlano [14]. 
For $f \in C^{1}\left(0, T_{f}, L^{2}\left(\Omega, \mathbb{R}^{2}\right)\right)$ and $g \in C^{1}\left(0, T_{f}, L^{2}\left(\partial \Omega_{N}, \mathbb{R}^{2}\right)\right)$, the external forces at time $t \in\left[0, T_{f}\right]$ with $T_{f}>0$ are collected into a functional $l(t) \in\left(H_{D}^{1}\right)^{*}$, where $\left(H_{D}^{1}\right)^{*}$ denotes the dual of $H_{D}^{1}$ :

$$
\langle l(t), \varphi\rangle:=\int_{\Omega} f(t) \cdot \varphi \mathrm{d} x+\int_{\partial \Omega_{N}} g(t) \cdot \varphi \mathrm{d} s \quad \forall \varphi \in H_{D}^{1} .
$$

In the following framework, we approximate the continuous-time visco-elasto-plastic evolution via discrete-time evolutions obtained by solving incremental variational problems. Given $T_{f}>0$ and a positive integer $N_{f}$, at each discrete time $t_{i}=i h, i=1, \ldots, N_{f}$, with $h=\frac{T_{f}}{N_{f}}$, let us assume that the approximate visco-elasto-plastic evolution $\left(u\left(t_{i-1}\right), v\left(t_{i-1}\right), e\left(t_{i-1}\right), p\left(t_{i-1}\right)\right)$ is known at $t_{i-1}$. We then define $\left(u\left(t_{i}\right), v\left(t_{i}\right), e\left(t_{i}\right), p\left(t_{i}\right)\right)$ as follows: $\left(u\left(t_{i}\right), e\left(t_{i}\right), p\left(t_{i}\right)\right)$ is defined at time $t_{i}$ as a minimizer of a deformation energy:

$$
\begin{aligned}
\mathcal{E}_{\text {def }}\left(u, v\left(t_{i-1}\right), e, p\right)= & \mathcal{E}_{e l}\left(e, v\left(t_{i-1}\right)\right)+\mathcal{E}_{v e}\left(u, u\left(t_{i-1}\right)\right) \\
& +\mathcal{E}_{p}\left(p, p\left(t_{i-1}\right)\right)-\left\langle l\left(t_{i}\right), u\right\rangle,
\end{aligned}
$$

with $v\left(t_{i-1}\right)$ fixed and among all $(u, e, p)$ triplets satisfying the kinematic admissibility condition. Then $v\left(t_{i}\right)$ is determined as a minimizer of the following variational problem:

$$
v\left(t_{i}\right):=\underset{v \leq v\left(t_{i-1}\right)}{\operatorname{argmin}} \mathcal{E}_{e l}\left(e\left(t_{i}\right), v\right)+\mathcal{E}_{S}(v) .
$$

In the following section, we describe a continuous time evolution of the proposed model.

\subsection{The visco-elasto-plastic evolution with regularized fracture}

We assume that the stress $\sigma=a A e+\beta E \dot{u}$ is the sum of two terms. The first term represents the stress associated to elastic deformation. It is affected by fracture via the factor $a(x, t)$. The second term represents the effect of viscous deformation. Let $u \in W^{1, \infty}\left(0, T_{f}, H^{1}\left(\Omega ; \mathbb{R}^{2}\right)\right), v \in W^{1, \infty}\left(0, T_{f}, H^{1}(\Omega ; \mathbb{R})\right)$, $e \in W^{1, \infty}\left(0, T_{f}, L^{2}\left(\Omega ; \mathbb{M}_{\text {sym }}^{2 \times 2}\right)\right), p \in W^{1, \infty}\left(0, T_{f}, L^{2}\left(\Omega ; \mathbb{M}_{\text {sym }}^{2 \times 2}\right)\right)$. We call

$$
(u, v, e, p): \Omega \times\left[0, T_{f}\right] \longrightarrow \mathbb{R}^{2} \times \mathbb{R} \times \mathbb{M}_{\mathrm{sym}}^{2 \times 2} \times \mathbb{M}_{\mathrm{sym}}^{2 \times 2}
$$

a continuous evolution if it satisfies the following properties:

- (H1) Initial conditions: $(u(0), v(0), e(0), p(0))=\left(u_{0}, v_{0}, e_{0}, p_{0}\right)$ with

$\left(u_{0}, e_{0}, p_{0}\right) \in A_{a d m}$, and $v_{0} \in H^{1}(\Omega)$ with $v_{0}=1$ on $\partial \Omega_{D}$ and $0 \leq v_{0} \leq 1$ a.e. in $\Omega$. We suppose also $\left(v_{0}^{2}+\eta\right)\left|A e_{0}\right| \leq \tau$.

- (H2) for every $t \in\left[0, T_{f}\right], v(t)=1$ on $\partial \Omega_{D}$, and $0 \leq v(t) \leq 1$ a.e. in $\Omega$,

- (H3) Irreversibility: for a.e. $t \in\left[0, T_{f}\right], \dot{v}(t) \leq 0$ a.e. in $\Omega$,

- (H4) Kinematic compatibility: for every $t \in\left[0, T_{f}\right], v(t)=1$ on $\partial \Omega_{D}$,

$$
E u(t)=e(t)+p(t) \text { a.e. in } \Omega \text { and } u(t)=0 \text { a.e. on } \partial \Omega_{D} .
$$

- (H5) Equilibrium condition: for a.e. $t \in\left[0, T_{f}\right]$,

$$
\begin{aligned}
& -\operatorname{div}(\sigma(t))=f(t), \quad \text { a.e. in } \quad \Omega, \\
& \sigma(t) \cdot \boldsymbol{n}=g(t), \quad \text { a.e. on } \quad \partial \Omega_{N},
\end{aligned}
$$

where $\sigma(t)=a(t) A e(t)+\beta E \dot{u}(t)$ and $a(t)=(v(t))^{2}+\eta$.

- (H6) Plastic flow rule: for a.e. $t \in\left[0, T_{f}\right]$,

$$
a(t) A e(t) \in \partial H(\dot{p}(t)) \text { for a.e. } \quad x \in \Omega .
$$

- (H7) Crack propagation condition: for a.e. $t \in\left[0, T_{f}\right]$,

$$
\mathcal{E}_{e l}(e(t), v(t))+\mathcal{E}_{S}(v(t))=\inf _{v \in H^{1}(\Omega), v=1 \text { on } \partial \Omega_{D}, v \leq v(t)} \mathcal{E}_{e l}(e(t), v)+\mathcal{E}_{S}(v) .
$$




\section{EXISTENCE RESUlT}

The main result of the paper is the existence result for the visco-elasto-plastic model with fracture.

Theorem 3.1. Let $\beta>0, \tau>0, \varepsilon>0, \eta>0$. We suppose that $v_{0}$ satisfies the crack propagation condition (H7) with $e_{0}$ i.e.

$$
\mathcal{E}_{e l}\left(e_{0}, v_{0}\right)+\mathcal{E}_{S}\left(v_{0}\right)=\inf _{v \in H^{1}(\Omega), v=1 \text { on } \partial \Omega_{D}, v \leq v_{0}} \mathcal{E}_{e l}\left(e_{0}, v\right)+\mathcal{E}_{S}(v) .
$$

Then, there exists at least one solution

$$
\left\{\begin{array}{l}
u \in W^{1, \infty}\left(0, T_{f}, H^{1}\left(\Omega ; \mathbb{R}^{2}\right)\right), \\
v \in W^{1, \infty}\left(0, T_{f}, H^{1}(\Omega ; \mathbb{R})\right), \\
e \in W^{1, \infty}\left(0, T_{f}, L^{2}\left(\Omega ; \mathbb{M}_{\text {sym }}^{2 \times 2}\right)\right), \\
p \in W^{1, \infty}\left(0, T_{f}, L^{2}\left(\Omega ; \mathbb{M}_{\text {sym }}^{2 \times 2}\right)\right),
\end{array}\right.
$$

satisfying $(\mathrm{H} 1)-(\mathrm{H} 7)$.

\subsection{Time discretization}

The proof of Theorem 3.1 is based on a time discretization procedure. We consider a partition of the time interval $\left[0, T_{f}\right]$ into $N_{f}$ sub-intervals of equal length $h$ :

$$
0=t_{h}^{0}<t_{h}^{1}<\ldots<t_{h}^{n}=n h<\ldots<t_{h}^{N_{f}}=T_{f}, \text { with } \quad h=\frac{T_{f}}{N_{f}}=t_{h}^{n}-t_{h}^{n-1} \rightarrow 0 .
$$

We set $v_{h}^{0}=v_{0}, u_{h}^{0}=u_{0}, e_{h}^{0}=e_{0}, p_{h}^{0}=p_{0}$. Note that in the whole text, $C>0$ denotes a generic constant which is independent of the discretization parameters. For $n=1, \ldots, N_{f}, N_{f} \geq 2$, we construct $\left(u_{h}^{n}, v_{h}^{n}, e_{h}^{n}, p_{h}^{n}\right)$ using an alternate minimization procedure. We define the deformation energy

$$
\begin{aligned}
\mathcal{E}_{d e f}\left(z, v_{h}^{n-1}, \xi, q\right)= & \frac{1}{2} \int_{\Omega} a_{h}^{n-1} A \xi: \xi \mathrm{d} x+\frac{1}{2 h} \beta\left\|E z-E u_{h}^{n-1}\right\|_{L^{2}}^{2} \\
& +\tau \int_{\Omega}\left|q-p_{h}^{n-1}\right| \mathrm{d} x-\left\langle l\left(t_{h}^{n}\right), z\right\rangle \\
= & \mathcal{E}_{e l}\left(v_{h}^{n-1}, \xi\right)+\mathcal{E}_{v e}\left(z, u_{h}^{n-1}\right)+\mathcal{E}_{p}\left(q, p_{h}^{n-1}\right)-\left\langle l\left(t_{h}^{n}\right), z\right\rangle,
\end{aligned}
$$

with $a_{h}^{n-1}:=\left[v_{h}^{n-1}\right]^{2}+\eta, \beta>0, \tau>0$. Since $\mathcal{E}_{\text {def }}\left(., v_{h}^{n-1}, .,.\right)$ is strictly convex, coercive and $A_{a d m}$ is a convex closed set, we have

Proposition 3.2. Suppose that $\left(u_{h}^{n-1}, e_{h}^{n-1}, p_{h}^{n-1}\right) \in A_{a d m}$. There exists unique minimizer to the variational problem

$$
\min _{(z, \xi, q) \in A_{\text {adm }}} \mathcal{E}_{\text {def }}\left(z, v_{h}^{n-1}, \xi, q\right) .
$$

We now define $\left(u_{h}^{n}, e_{h}^{n}, p_{h}^{n}\right)$ as a solution of (3.1) and we derive the Euler-Lagrange equation satisfied by this solution.

Proposition 3.3. Let $\left(u_{h}^{n}, e_{h}^{n}, p_{h}^{n}\right) \in A_{a d m}$ be a solution to (3.1) with

$$
\sigma_{h}^{n}:=a_{h}^{n-1} A e_{h}^{n}+\beta \frac{E u_{h}^{n}-E u_{h}^{n-1}}{h}=a_{h}^{n-1} A e_{h}^{n}+\beta E \delta u_{h}^{n}
$$


Then, for all $n \in\left\{1, \ldots, N_{f}\right\}$ :

$$
\begin{cases}-\operatorname{div}\left(\sigma_{h}^{n}\right)=f\left(t_{h}^{n}\right), & \text { a.e. in } \Omega, \\ \sigma_{h}^{n} \cdot \boldsymbol{n}=g\left(t_{h}^{n}\right), & \text { a.e on } \partial \Omega_{N}, \\ a_{h}^{n-1} A e_{h}^{n} \in \partial H\left(p_{h}^{n}-p_{h}^{n-1}\right) & \text { a.e. in } \Omega .\end{cases}
$$

Proof. Let $(z, \xi, q) \in A_{a d m}$, then $\left(u_{h}^{n}+s z, e_{h}^{n}+s \xi, p_{h}^{n}+s q\right) \in A_{a d m}$ is an admissible triplet for every $0<s<1$. We have

$$
\mathcal{E}_{\text {def }}\left(u_{h}^{n}, v_{h}^{n-1}, e_{h}^{n}, p_{h}^{n}\right) \leq \mathcal{E}_{\text {def }}\left(u_{h}^{n}+s z, v_{h}^{n-1}, e_{h}^{n}+s \xi, p_{h}^{n}+s q\right),
$$

hence

$$
\begin{aligned}
0 \leq & s \int_{\Omega} a_{h}^{n-1} A e_{h}^{n}: \xi \mathrm{d} x+s \int_{\Omega} \beta \frac{E u_{h}^{n}-E u_{h}^{n-1}}{h}:(\xi+q) \mathrm{d} x \\
& +\tau \int_{\Omega}\left|p_{h}^{n}+s q-p_{h}^{n-1}\right|-\left|p_{h}^{n}-p_{h}^{n-1}\right| \mathrm{d} x-s\left\langle l\left(t_{h}^{n}\right), z\right\rangle+o(s) .
\end{aligned}
$$

Let $\Psi(s):=\tau \int_{\Omega}\left|p_{h}^{n}+s q-p_{h}^{n-1}\right| \mathrm{d} x$. Using the convexity of $\Psi$ we have $\Psi(s)-\Psi(0) \leq s(\Psi(1)-\Psi(0))$. Dividing this inequality by $\mathrm{s}$ and letting $\mathrm{s}$ tend to zero implies that

$$
\begin{aligned}
& \int_{\Omega} a_{h}^{n-1} A e_{h}^{n}: \xi \mathrm{d} x+\beta \int_{\Omega} \frac{E u_{h}^{n}-E u_{h}^{n-1}}{h}:(\xi+q) \mathrm{d} x \\
& +\tau \int_{\Omega}\left|p_{h}^{n}-p_{h}^{n-1}+q\right|-\left|p_{h}^{n}-p_{h}^{n-1}\right| \mathrm{d} x \\
\geqslant & \left\langle l\left(t_{h}^{n}\right), z\right\rangle .
\end{aligned}
$$

Testing (3.3) with $(z, \xi, q)= \pm(\phi, E \phi, 0)$ for any $\phi \in C_{c}^{\infty}\left(\Omega, \mathbb{R}^{2}\right)$, we obtain

$$
\int_{\Omega} \sigma_{h}^{n}: E(\phi) \mathrm{d} x=\left\langle l\left(t_{h}^{n}\right), \phi\right\rangle
$$

and $-\operatorname{div}\left(\sigma_{h}^{n}\right)=f\left(t_{h}^{n}\right)$ a.e. in $\Omega$. Furher, picking $\phi \in C^{\infty}\left(\bar{\Omega}, \mathbb{R}^{2}\right)$, with $\phi=0$ on $\partial \Omega_{D}$ in $\pm(\phi, E \phi, 0)$ as a test function for (3.3) and integrating (3.4) by parts, we also obtain that $\sigma_{h}^{n} \cdot \boldsymbol{n}=g\left(t_{h}^{n}\right)$ a.e. on $\partial \Omega_{N}$. Testing (3.3) with $\left(0,-q+p_{h}^{n}-p_{h}^{n-1}, q-p_{h}^{n}+p_{h}^{n-1}\right)$ for any $q \in L^{2}\left(\Omega, \mathbb{M}_{\mathrm{sym}}^{2 \times 2}\right)$, we have

$$
\tau \int_{\Omega}|q| \mathrm{d} x \geq \tau \int_{\Omega}\left|p_{h}^{n}-p_{h}^{n-1}\right| \mathrm{d} x+\int_{\Omega} a_{h}^{n-1} A e_{h}^{n}:\left(q-\left(p_{h}^{n}-p_{h}^{n-1}\right)\right) \mathrm{d} x,
$$

so that

$$
\tau|q| \geq \tau\left|p_{h}^{n}(x)-p_{h}^{n-1}(x)\right|+a_{h}^{n-1}(x) A e_{h}^{n}(x):\left(q-\left(p_{h}^{n}(x)-p_{h}^{n-1}(x)\right)\right)
$$

for all $q \in \mathbb{M}_{\text {sym }}^{2 \times 2}$ and for a.e. $x \in \Omega$, which by definition of the subdifferential implies that

$$
a_{h}^{n-1} A e_{h}^{n} \in \partial H\left(p_{h}^{n}-p_{h}^{n-1}\right) \quad \text { a.e.in } \Omega .
$$

Proposition 3.4. For given $e_{h}^{n} \in L^{2}\left(\Omega, \mathbb{M}_{\text {sym }}^{2 \times 2}\right)$ there exists an unique minimizer $v_{h}^{n}$ to

$$
v_{h}^{n}:=\underset{v \in H^{1}(\Omega), v=1 \text { on } \partial \Omega_{D}, v \leq v_{h}^{n-1}}{\operatorname{argmin}}\left\{\mathcal{E}_{e l}\left(e_{h}^{n}, v\right)+\mathcal{E}_{S}(v)\right\} .
$$


Additionally, for all $n \in\left\{1, \ldots, N_{f}\right\}, v_{h}^{n}$ satisfies the following variational inequality:

$$
\begin{aligned}
2 \varepsilon \int_{\Omega} \nabla v_{h}^{n} \nabla\left(v_{h}^{n}-\varphi\right) \mathrm{d} x & +\int_{\Omega} v_{h}^{n} A e_{h}^{n}: e_{h}^{n}\left(v_{h}^{n}-\varphi\right) \mathrm{d} x \\
& +(2 \varepsilon)^{-1} \int_{\Omega}\left(v_{h}^{n}-1\right)\left(v_{h}^{n}-\varphi\right) \mathrm{d} x \leq 0,
\end{aligned}
$$

for any $\varphi \in H^{1}(\Omega), \varphi=1$ on $\partial \Omega_{D}$ and $\varphi \leq v_{h}^{n-1}$. Furthermore, $v_{h}^{n}$ satisfies the comparison principle $0 \leq v_{h}^{n} \leq$ $v_{h}^{n-1}$ a.e. in $\Omega$.

Proof. The existence and uniqueness of the solution of (3.7) follow from the strict convexity and coercivity of the functional $\mathcal{E}_{e l}\left(e_{h}^{n},.\right)+\mathcal{E}_{S}($.$) , and since \left\{v \in H^{1}(\Omega), v=1\right.$ on $\left.\partial \Omega_{D}, v \leq v_{h}^{n-1}\right\}$ is a closed convex set. Let $\varphi$ an admissible function for (3.7), then $\psi=v_{h}^{n}+t\left(\varphi-v_{h}^{n}\right)$ with $0<t<1$ is an admissible function for (3.7). In fact, $\psi \in H^{1}(\Omega), \psi=1$ on $\partial \Omega_{D}$ and

$$
\begin{aligned}
\psi & \leq v_{h}^{n}+t\left(v_{h}^{n-1}-v_{h}^{n}\right)=v_{h}^{n}(1-t)+t v_{h}^{n-1} \\
& \leq v_{h}^{n-1}(1-t)+t v_{h}^{n-1}=v_{h}^{n-1} .
\end{aligned}
$$

By definition of $v_{h}^{n}, \mathcal{E}_{e l}\left(e_{h}^{n}, v_{h}^{n}\right)+\mathcal{E}_{S}\left(v_{h}^{n}\right) \leq \mathcal{E}_{e l}\left(e_{h}^{n}, \psi\right)+\mathcal{E}_{S}(\psi)$. We obtain:

$$
2 \varepsilon \int_{\Omega} \nabla v_{h}^{n} \nabla\left(v_{h}^{n}-\varphi\right) \mathrm{d} x+\int_{\Omega} v_{h}^{n} A e_{h}^{n}: e_{h}^{n}\left(v_{h}^{n}-\varphi\right) \mathrm{d} x+(2 \varepsilon)^{-1} \int_{\Omega}\left(v_{h}^{n}-1\right)\left(v_{h}^{n}-\varphi\right) \mathrm{d} x \leq 0 .
$$

Testing (3.9) with $\varphi=\max \left(0, v_{h}^{n}\right)$ gives

$$
2 \varepsilon \int_{\left\{v_{h}^{n} \leq 0\right\}} \nabla v_{h}^{n} \nabla v_{h}^{n} \mathrm{~d} x+\int_{\left\{v_{h}^{n} \leq 0\right\}}\left(v_{h}^{n}\right)^{2} A e_{h}^{n}: e_{h}^{n} \mathrm{~d} x+(2 \varepsilon)^{-1} \int_{\left\{v_{h}^{n} \leq 0\right\}}\left(v_{h}^{n}-1\right) v_{h}^{n} \mathrm{~d} x \leq 0,
$$

so that $v_{h}^{n}=0$ a.e. on $\left\{v_{h}^{n} \leq 0\right\}$. It follows that $v_{h}^{n} \geqslant 0$ a.e. in $\Omega$.

Remark 3.5. Testing (3.9) with $\varphi=v_{h}^{n-1}$ and with $\varphi=2 v_{h}^{n}-v_{h}^{n-1}$ we derive the equality

$$
2 \varepsilon \int_{\Omega} \nabla v_{h}^{n} \nabla\left(v_{h}^{n}-v_{h}^{n-1}\right) \mathrm{d} x+\int_{\Omega} v_{h}^{n} A e_{h}^{n}: e_{h}^{n}\left(v_{h}^{n}-v_{h}^{n-1}\right) \mathrm{d} x+(2 \varepsilon)^{-1} \int_{\Omega}\left(v_{h}^{n}-1\right)\left(v_{h}^{n}-v_{h}^{n-1}\right) \mathrm{d} x=0 .
$$

\subsection{A priori estimates}

We define for all $n \geqslant 1$,

$$
\delta u_{h}^{n}:=\frac{u_{h}^{n}-u_{h}^{n-1}}{h}, \quad \delta v_{h}^{n}:=\frac{v_{h}^{n}-v_{h}^{n-1}}{h}, \quad \delta e_{h}^{n}:=\frac{e_{h}^{n}-e_{h}^{n-1}}{h}, \quad \delta p_{h}^{n}:=\frac{p_{h}^{n}-p_{h}^{n-1}}{h} .
$$

Proposition 3.6. There exists a constant $C>0$ independent of $h$ and $n$ such that

$$
\max _{\left\{1, . . N_{f}\right\}}\left(\left\|\delta u_{h}^{n}\right\|_{H^{1}},\left\|\delta v_{h}^{n}\right\|_{H^{1}},\left\|\delta e_{h}^{n}\right\|_{L^{2}},\left\|\delta p_{h}^{n}\right\|_{L^{2}}\right) \leq C .
$$

For the proof of Proposition 3.6 we need following lemma.

Lemma 3.7. For all $n \geqslant 1$, we have $a_{h}^{n-1} A e_{h}^{n} \in K$ a.e. in $\Omega$. Additionally, there exists a constant $C>0$, independent of $h$ and $n$ such that

$$
\left\|A e_{h}^{n}\right\|_{L^{\infty}} \leq C .
$$


Proof. Testing (3.5) with $q^{\prime}=a_{h}^{n-1} A e_{h}^{n}(x)+p_{h}^{n}(x)-p_{h}^{n-1}(x)$ leads to $\left|a_{h}^{n-1} A e_{h}^{n}(x)\right| \leq \tau$ for a.e. $x \in \Omega$. Since $\eta \leq a_{h}^{n-1}(x)$ for all $x \in \Omega$, we have $\left\|A e_{h}^{n}\right\|_{L^{\infty}} \leq C$.

Lemma 3.8. For all $q \in K=\left\{q^{*} \in \mathbb{M}_{\text {sym }}^{2 \times 2} ;\left|q^{*}\right| \leq \tau\right\}$ and $n \geqslant 1$, we have

$$
a_{h}^{n-1} A e_{h}^{n}: \delta p_{h}^{n} \geqslant q: \delta p_{h}^{n}, \quad \text { a.e. in } \Omega .
$$

Proof. By convex duality

$$
a_{h}^{n-1} A e_{h}^{n} \in \partial H\left(p_{h}^{n}-p_{h}^{n-1}\right) \Longleftrightarrow p_{h}^{n}-p_{h}^{n-1} \in \partial H^{*}\left(a_{h}^{n-1} A e_{h}^{n}\right)
$$

where $H^{*}\left(q^{*}\right)=1_{K}\left(q^{*}\right)$ denotes the convex conjugate of H. Since $a_{h}^{n-1} A e_{h}^{n} \in K$ a.e. in $\Omega$, we have for all $q \in K$ $a_{h}^{n-1} A e_{h}^{n}: \delta p_{h}^{n} \geqslant q: \delta p_{h}^{n}$ a.e. in $\Omega$.

Lemma 3.9. For all $n \in\left\{1, \ldots, N_{f}\right\}$, there exists a constant $C>0$ independent of $h$ and $n$ such that

$$
\left\|\delta v_{h}^{n}\right\|_{L^{2}}^{2}+\left\|\nabla \delta v_{h}^{n}\right\|_{L^{2}}^{2} \leq C\left\|\delta e_{h}^{n}\right\|_{L^{2}}^{2} .
$$

Proof. We proceed as in the proof of Lemma 3.4 in [16]. We write inequality (3.9) at time $t_{h}^{n-1}$ :

$$
\begin{aligned}
& 2 \varepsilon \int_{\Omega} \nabla v_{h}^{n-1} \nabla\left(v_{h}^{n-1}-\varphi\right) \mathrm{d} x+\int_{\Omega} v_{h}^{n-1} A e_{h}^{n-1}: e_{h}^{n-1}\left(v_{h}^{n-1}-\varphi\right) \mathrm{d} x \\
+ & (2 \varepsilon)^{-1} \int_{\Omega}\left(v_{h}^{n-1}-1\right)\left(v_{h}^{n-1}-\varphi\right) \mathrm{d} x \leq 0
\end{aligned}
$$

for any $\varphi \leq v_{h}^{n-2}, \varphi \in H^{1}(\Omega), \varphi=1$ on $\partial \Omega_{D}$. We choose $\varphi=v_{h}^{n} \leq v_{h}^{n-2}$ and divide (3.16) by $h$ :

$$
\begin{aligned}
& 2 \varepsilon \int_{\Omega} \nabla v_{h}^{n-1} \nabla \delta v_{h}^{n} \mathrm{~d} x+\int_{\Omega} v_{h}^{n-1} A e_{h}^{n-1}: e_{h}^{n-1} \delta v_{h}^{n} \mathrm{~d} x \\
+ & (2 \varepsilon)^{-1} \int_{\Omega}\left(v_{h}^{n-1}-1\right) \delta v_{h}^{n} \mathrm{~d} x \geqslant 0 .
\end{aligned}
$$

We divide (3.11) by $h$ and substract (3.17). Then we use the equality $a^{2}-b^{2}=(a-b)^{2}+2(a-b) b$, the fact that $\delta v_{h}^{n} \leq 0$, and the Cauchy-Schwarz inequality to obtain

$$
\begin{aligned}
\left\|\delta v_{h}^{n}\left|e_{h}^{n}\right|_{A}\right\|_{L^{2}}^{2}+ & (2 \varepsilon)^{-1}\left\|\delta v_{h}^{n}\right\|_{L^{2}}^{2}+(2 \varepsilon)\left\|\nabla \delta v_{h}^{n}\right\|_{L^{2}}^{2} \\
\leq & \frac{1}{h} \int_{\Omega}\left(\left|e_{h}^{n-1}\right|_{A}^{2}-\left|e_{h}^{n}\right|_{A}^{2}\right) v_{h}^{n-1} \delta v_{h}^{n} \mathrm{~d} x \\
= & \frac{1}{h} \int_{\Omega}\left|\left(e_{h}^{n-1}-e_{h}^{n}\right)\right|_{A}^{2} v_{h}^{n-1} \delta v_{h}^{n} \mathrm{~d} x \\
& +\frac{2}{h} \int_{\Omega} A\left(e_{h}^{n-1}-e_{h}^{n}\right): e_{h}^{n} v_{h}^{n-1} \delta v_{h}^{n} \mathrm{~d} x \\
\leq & \frac{2}{h} \int_{\Omega}\left(\left|e_{h}^{n}\right|_{A}\left|\delta v_{h}^{n}\right|\right)\left(\left|v_{h}^{n-1} \|\left(e_{h}^{n-1}-e_{h}^{n}\right)\right|_{A}\right) \mathrm{d} x \\
\leq & 2\left\|\left(\delta v_{h}^{n}\right)\left|e_{h}^{n}\right|_{A}\right\|_{L^{2}}\left\|v_{h}^{n-1}\left|\delta e_{h}^{n}\right|_{A}\right\|_{L^{2}} .
\end{aligned}
$$

The result follows from the Young inequality $a b \leq a^{2} / 2+b^{2} / 2$.

We now prove Proposition 3.6. 
Proof. Testing (3.3) with $(z, \xi, q)= \pm\left(h \delta u_{h}^{n}, E\left(h \delta u_{h}^{n}\right), 0\right)$ where $E\left(h \delta u_{h}^{n}\right)=h\left(\delta e_{h}^{n}+\delta p_{h}^{n}\right)$, we obtain

$$
\begin{aligned}
& \int_{\Omega} a_{h}^{n-1} A e_{h}^{n}:\left(e_{h}^{n}-e_{h}^{n-1}\right) \mathrm{d} x+\int_{\Omega} a_{h}^{n-1} A e_{h}^{n}:\left(p_{h}^{n}-p_{h}^{n-1}\right) \mathrm{d} x+h \beta\left\|E \delta u_{h}^{n}\right\|_{L^{2}}^{2} \\
& =\left\langle l\left(t_{h}^{n}\right), u_{h}^{n}-u_{h}^{n-1}\right\rangle, \\
& \int_{\Omega} a_{h}^{n-1} A e_{h}^{n}:\left(p_{h}^{n}-p_{h}^{n-1}\right) \mathrm{d} x= \\
& \quad\left\langle l\left(t_{h}^{n}\right), u_{h}^{n}-u_{h}^{n-1}\right\rangle-h \beta\left\|E \delta u_{h}^{n}\right\|_{L^{2}}^{2} \\
& \quad-\int_{\Omega} a_{h}^{n-1} A e_{h}^{n}:\left(e_{h}^{n}-e_{h}^{n-1}\right) \mathrm{d} x .
\end{aligned}
$$

Testing (3.3) with $\left(0, h \delta p_{h}^{n},-h \delta p_{h}^{n}\right)$ yields

$$
\int_{\Omega} a_{h}^{n-1} A e_{h}^{n}:\left(p_{h}^{n}-p_{h}^{n-1}\right) \mathrm{d} x \geqslant \tau \int_{\Omega}\left|p_{h}^{n}-p_{h}^{n-1}\right| \mathrm{d} x .
$$

Combining (3.19) and (3.20), we get

$$
\begin{aligned}
& \int_{\Omega} a_{h}^{n-1} A e_{h}^{n}:\left(e_{h}^{n}-e_{h}^{n-1}\right) \mathrm{d} x+h \beta\left\|E \delta u_{h}^{n}\right\|_{L^{2}}^{2}+\tau \int_{\Omega}\left|p_{h}^{n}-p_{h}^{n-1}\right| \mathrm{d} x \\
\leqslant & \left\langle l\left(t_{h}^{n}\right), u_{h}^{n}-u_{h}^{n-1}\right\rangle .
\end{aligned}
$$

The first term on the left-hand side can be analysed in a similar way as in [16]:

$$
\begin{aligned}
& \int_{\Omega} a_{h}^{n-1} A e_{h}^{n}:\left(e_{h}^{n}-e_{h}^{n-1}\right) \mathrm{d} x=\mathcal{E}_{e l}\left(e_{h}^{n}, v_{h}^{n}\right)-\mathcal{E}_{e l}\left(e_{h}^{n-1}, v_{h}^{n-1}\right) \\
& +\frac{1}{2} h^{2}\left\|\left(a_{h}^{n-1}\right)^{1 / 2}\left|\delta e_{h}^{n}\right|_{A}\right\|_{L^{2}}^{2}-\frac{1}{2} \int_{\Omega}\left(a_{h}^{n}-a_{h}^{n-1}\right)\left|e_{h}^{n}\right|_{A}^{2} \mathrm{~d} x .
\end{aligned}
$$

Further, we observe that

$$
a_{h}^{n}-a_{h}^{n-1}=\left(v_{h}^{n}\right)^{2}-\left(v_{h}^{n-1}\right)^{2}=h\left(v_{h}^{n}+v_{h}^{n-1}\right) \delta v_{h}^{n}=2 h v_{h}^{n} \delta v_{h}^{n}-h^{2}\left|\delta v_{h}^{n}\right|^{2} .
$$

Thanks to (3.11), we obtain

$$
\begin{aligned}
-\frac{1}{2} \int_{\Omega}\left(a_{h}^{n}-a_{h}^{n-1}\right)\left|e_{h}^{n}\right|_{A}^{2} \mathrm{~d} x= & (2 \varepsilon)^{-1} \int_{\Omega}\left(v_{h}^{n}-1\right)\left(v_{h}^{n}-v_{h}^{n-1}\right) \mathrm{d} x \\
& +2 \varepsilon \int_{\Omega} \nabla v_{h}^{n}\left(\nabla v_{h}^{n}-\nabla v_{h}^{n-1}\right) \mathrm{d} x+\frac{1}{2} h^{2}\left\|\left(\delta v_{h}^{n}\right)\left|e_{h}^{n}\right|_{A}\right\|_{L^{2}}^{2},
\end{aligned}
$$

and rewriting $v_{h}^{n}-v_{h}^{n-1}=\left(v_{h}^{n}-1\right)-\left(v_{h}^{n-1}-1\right)$, yields

$$
\begin{aligned}
& -\frac{1}{2} \int_{\Omega}\left(a_{h}^{n}-a_{h}^{n-1}\right)\left|e_{h}^{n}\right|_{A}^{2} \mathrm{~d} x=\mathcal{E}_{S}\left(v_{h}^{n}\right)-\mathcal{E}_{S}\left(v_{h}^{n-1}\right) \\
& +h^{2}\left((4 \varepsilon)^{-1}\left\|\delta v_{h}^{n}\right\|_{L^{2}}^{2}+\varepsilon\left\|\nabla \delta v_{h}^{n}\right\|_{L^{2}}^{2}\right)+\frac{1}{2} h^{2}\left\|\delta v_{h}^{n}\left|e_{h}^{n}\right|_{A}\right\|_{L^{2}}^{2} .
\end{aligned}
$$

Summing (3.21) for $1 \leq n \leq N$ and using (3.22) and (3.23) we obtain

$$
\begin{aligned}
& \mathcal{E}_{e l}\left(e_{h}^{N}, v_{h}^{N}\right)+\mathcal{E}_{S}\left(v_{h}^{N}\right)+\sum_{n=1}^{N} h \beta\left\|E \delta u_{h}^{n}\right\|_{L^{2}}^{2}+\sum_{n=1}^{N} h \int_{\Omega}\left|\delta p_{h}^{n}\right| \mathrm{d} x \\
\leq & \sum_{n=1}^{N}\left\langle l\left(t_{h}^{n}\right), u_{h}^{n}-u_{h}^{n-1}\right\rangle+\mathcal{E}_{e l}\left(e_{0}, v_{0}\right)+\mathcal{E}_{S}\left(v_{0}\right),
\end{aligned}
$$


where $1 \leq N \leq N_{f}$. Using the Korn inequality we obtain the following estimate

$$
\begin{aligned}
\sum_{n=1}^{N} h\left\langle l\left(t_{h}^{n}\right), \delta u_{h}^{n}\right\rangle & \leq\left(\sum_{n=1}^{N} h\left\|l\left(t_{h}^{n}\right)\right\|_{\left(H_{D}^{1}\right)^{*}}^{2}\right)^{1 / 2}\left(\sum_{n=1}^{N} h\left\|\nabla \delta u_{h}^{n}\right\|_{L^{2}}^{2}\right)^{1 / 2} \\
& \leq C\left(\sum_{n=1}^{N} h\left\|l\left(t_{h}^{n}\right)\right\|_{\left(H_{D}^{1}\right)^{*}}^{2}\right)^{1 / 2}\left(\sum_{n=1}^{N} h\left\|E \delta u_{h}^{n}\right\|_{L^{2}}^{2}\right)^{1 / 2} .
\end{aligned}
$$

For all $N \in\left\{1, \ldots, N_{f}\right\}$ yields

$$
\left\|e_{h}^{N}\right\|_{2}^{2}+\left\|v_{h}^{N}\right\|_{L^{2}}^{2}+\left\|\nabla v_{h}^{N}\right\|_{L^{2}}^{2}+\sum_{n=1}^{N} h \beta\left\|E \delta u_{h}^{n}\right\|_{2}^{2}+\sum_{n=1}^{N} h \int_{\Omega}\left|\delta p_{h}^{n}\right| \mathrm{d} x \leq C,
$$

where $C>0$ is a constant independent of $h$ and $n$. Let $N_{0}$ such that $\left\{\left\|e_{h}^{N_{0}}\right\|_{2}^{2}+\left\|v_{h}^{N_{0}}\right\|_{L^{2}}^{2}+\left\|\nabla v_{h}^{N_{0}}\right\|_{L^{2}}^{2}\right\}$ is maximal between $1 \leq N \leq N_{f}$. Inequality (3.24) is true for $N=N_{0}$, and $N=N_{f}$. Thus,

$$
\max _{1 \leq n \leq N_{f}}\left\{\left\|e_{h}^{n}\right\|_{2}^{2}+\left\|v_{h}^{n}\right\|_{L^{2}}^{2}+\left\|\nabla v_{h}^{n}\right\|_{L^{2}}^{2}\right\}+\sum_{n=1}^{N_{f}} h \beta\left\|E \delta u_{h}^{n}\right\|_{2}^{2}+\sum_{n=1}^{N_{f}} h \int_{\Omega}\left|\delta p_{h}^{n}\right| \mathrm{d} x \leq 2 C .
$$

Further, using the inequality $a_{h}^{n-1} \leq 1+\eta$, for all $n \in\left\{1, \ldots, N_{f}\right\}$ we obtain from Proposition 3.3

$$
\begin{aligned}
\left\|\sigma_{h}^{n}\right\|_{L^{2}}^{2} & =\int_{\Omega}\left|\sigma_{h}^{n}\right|^{2} \mathrm{~d} x=\int_{\Omega} \sigma_{h}^{n}: a_{h}^{n-1} A e_{h}^{n} \mathrm{~d} x+\beta \int_{\Omega} \sigma_{h}^{n}: E \delta u_{h}^{n} \mathrm{~d} x \\
& \leq C\left\|\sigma_{h}^{n}\right\|_{L^{2}}\left\|e_{h}^{n}\right\|_{L^{2}}+\beta\left\langle l\left(t_{h}^{n}\right), \delta u_{h}^{n}\right\rangle \\
& \leq C\left\|\sigma_{h}^{n}\right\|_{L^{2}}\left\|e_{h}^{n}\right\|_{L^{2}}+C\left\|l\left(t_{h}^{n}\right)\right\|_{\left(H_{D}^{1}\right)^{*}} \beta\left\|\nabla \delta u_{h}^{n}\right\|_{L^{2}} \\
& \leq C\left\|\sigma_{h}^{n}\right\|_{L^{2}}\left\|e_{h}^{n}\right\|_{L^{2}}+C\left\|l\left(t_{h}^{n}\right)\right\|_{\left(H_{D}^{1}\right) *}\left\|\beta E \delta u_{h}^{n}\right\|_{L^{2}} \\
& \leq C\left\|\sigma_{h}^{n}\right\|_{L^{2}}\left\|e_{h}^{n}\right\|_{L^{2}}+C\left\|l\left(t_{h}^{n}\right)\right\|_{\left(H_{D}^{1}\right)^{*}}\left(\left\|\sigma_{h}^{n}\right\|_{2}+\left\|e_{h}^{n}\right\|_{L^{2}}\right) .
\end{aligned}
$$

We thus deduce that

$$
\max _{\left\{1, . . N_{f}\right\}}\left\|\sigma_{h}^{n}\right\|_{L^{2}} \leq C
$$

It follows from (3.2), (3.25) and (3.27) that

$$
\max _{\left\{1, . . N_{f}\right\}}\left\|E \delta u_{h}^{n}\right\|_{L^{2}} \leq C .
$$

We now estimate $\delta e_{h}^{n}$ :

$$
\begin{aligned}
\eta \alpha_{1}\left\|\delta e_{h}^{n}\right\|_{L^{2}}^{2} & \leq \int_{\Omega} a_{h}^{n-1} A \delta e_{h}^{n}: \delta e_{h}^{n} \mathrm{~d} x \\
& =\int_{\Omega} a_{h}^{n-1} A \delta e_{h}^{n}: E \delta u_{h}^{n} \mathrm{~d} x-\int_{\Omega} a_{h}^{n-1} A \delta e_{h}^{n}: \delta p_{h}^{n} \mathrm{~d} x \\
& \leq C\left\|E \delta u_{h}^{n}\right\|_{L^{2}}\left\|\delta e_{h}^{n}\right\|_{L^{2}}-\int_{\Omega} a_{h}^{n-1} A \delta e_{h}^{n}: \delta p_{h}^{n} \mathrm{~d} x
\end{aligned}
$$

From Lemma 3.7 it follows that for $n \geqslant 2, a_{h}^{n-2} A e_{h}^{n-1} \in K$ a.e. in $\Omega$. This implies that $a_{h}^{n-1} A e_{h}^{n-1} \in K$ a.e. in $\Omega$ and from Lemma 3.8 we deduce that 
$\int_{\Omega} a_{h}^{n-1} A \delta e_{h}^{n}: \delta p_{h}^{n} \mathrm{~d} x \geqslant 0$, and thus $\left\|\delta e_{h}^{n}\right\|_{L^{2}} \leq C\left\|E \delta u_{h}^{n}\right\|_{L^{2}}$. Since $a_{h}^{0} A e_{h}^{0} \in K$, we also deduce from Lemma 3.8 that $\int_{\Omega} a_{h}^{0} A \delta e_{h}^{1}: \delta p_{h}^{1} \mathrm{~d} x \geqslant 0$, and thus $\left\|\delta e_{h}^{1}\right\|_{L^{2}} \leq C\left\|E \delta u_{h}^{1}\right\|_{L^{2}}$. By the estimate (3.28) and since $E \delta u_{h}^{n}=\delta e_{h}^{n}+\delta p_{h}^{n}$ we have

$$
\max _{\left\{1, . . N_{f}\right\}}\left(\left\|\delta e_{h}^{n}\right\|_{L^{2}},\left\|\delta p_{h}^{n}\right\|_{L^{2}}\right) \leq C .
$$

Applying Lemma 3.9 concludes the proof of Proposition 3.6.

\subsection{Compactness results}

We now define piecewise affine interpolants of the sequences $\left(u_{h}^{n}\right)_{n=0}^{N_{f}},\left(v_{h}^{n}\right)_{n=0}^{N_{f}},\left(e_{h}^{n}\right)_{n=0}^{N_{f}},\left(p_{h}^{n}\right)_{n=0}^{N_{f}}$ by

$$
\left(\begin{array}{l}
u_{h}(t) \\
v_{h}(t) \\
a_{h}(t) \\
e_{h}(t) \\
p_{h}(t)
\end{array}\right)=\left(\begin{array}{l}
u_{h}^{n} \\
v_{h}^{n} \\
a_{h}^{n} \\
e_{h}^{n} \\
p_{h}^{n}
\end{array}\right)+\left(t-t_{h}^{n}\right)\left(\begin{array}{l}
\delta u_{h}^{n} \\
\delta v_{h}^{n} \\
\delta a_{h}^{n} \\
\delta e_{h}^{n} \\
\delta p_{h}^{n}
\end{array}\right) \quad \text { for } t \in\left[t_{h}^{n-1}, t_{h}^{n}\right], \quad n=1, \ldots, N_{f}
$$

We define backward piecewise constant interpolant $u_{h}^{+}(., t)$ by

$$
u_{h}^{+}(t)=u_{h}^{n}, \quad \text { for } \quad t \in\left(t_{h}^{n-1}, t_{h}^{n}\right], \quad n=1, \ldots, N_{f}
$$

and similarly we define $v_{h}^{+}(t), e_{h}^{+}(t), p_{h}^{+}(t), l_{h}^{+}(t)$.

We also define $u_{h}^{+}(0)=u_{0}, v_{h}^{+}(0)=v_{0}, e_{h}^{+}(0)=e_{0}, p_{h}^{+}(0)=p_{0}$. The forward piecewise constant interpolant $a_{h}^{-}(., t)$ is defined by

$$
a_{h}^{-}(., t)=a_{h}^{n-1}, \quad \text { for } \quad t \in\left[t_{h}^{n-1}, t_{h}^{n}\right), \quad n=1, \ldots, N_{f} .
$$

Thanks to Proposition 3.6

$$
\begin{aligned}
& \left\|u_{h}\right\|_{W^{1, \infty}\left(0, T_{f}, H^{1}\left(\Omega, \mathbb{R}^{2}\right)\right)}+\left\|v_{h}\right\|_{W^{1, \infty}\left(0, T_{f}, H^{1}(\Omega, \mathbb{R})\right)} \\
& \quad+\left\|e_{h}\right\|_{W^{1, \infty}\left(0, T_{f}, L^{2}\left(\Omega, \mathbb{M}_{\mathrm{sym}}^{2 \times 2}\right)\right)}+\left\|p_{h}\right\|_{W^{1, \infty}\left(0, T_{f}, L^{2}\left(\Omega, \mathbb{M}_{\mathrm{sym}}^{2 \times 2}\right)\right)} \leq C .
\end{aligned}
$$

Hence, there exists a subsequence $h_{j} \searrow 0$ (we just write $\mathrm{h}$ ) and $u \in W^{1, \infty}\left(0, T_{f}, H^{1}\left(\Omega, \mathbb{R}^{2}\right)\right.$ ), $v \in$ $W^{1, \infty}\left(0, T_{f}, H^{1}(\Omega, \mathbb{R})\right)$, with $0 \leq v(t) \leq 1, v(t)=1$ on $\partial \Omega_{D}, \dot{v}(t) \leq 0$ a.e. in $\Omega$ for a.e. $t \in\left(0, T_{f}\right]$, $e \in W^{1, \infty}\left(0, T_{f}, L^{2}\left(\Omega, \mathbb{M}_{\mathrm{sym}}^{2 \times 2}\right)\right), p \in W^{1, \infty}\left(0, T_{f}, L^{2}\left(\Omega, \mathbb{M}_{\mathrm{sym}}^{2 \times 2}\right)\right)$ such that

$$
\begin{array}{lll}
u_{h}, \dot{u}_{h} \rightarrow u, \dot{u} & \text { weakly* in } & L^{\infty}\left(0, T_{f}, H^{1}\left(\Omega, \mathbb{R}^{2}\right)\right), \\
v_{h}, \dot{v}_{h} \rightarrow v, \dot{v} & \text { weakly* in } & L^{\infty}\left(0, T_{f}, H^{1}(\Omega, \mathbb{R})\right), \\
e_{h}, \dot{e}_{h} \rightarrow e, \dot{e} & \text { weakly* in } & L^{\infty}\left(0, T_{f}, L^{2}\left(\Omega, \mathbb{M}_{\mathrm{sym}}^{2 \times 2}\right)\right), \\
p_{h}, \dot{p}_{h} \rightarrow p, \dot{p} & \text { weakly* in } & L^{\infty}\left(0, T_{f}, L^{2}\left(\Omega, \mathbb{M}_{\mathrm{sym}}^{2 \times 2}\right)\right) .
\end{array}
$$

Using Arzelà-Ascoli Theorem and Proposition 3.6, we have for all $t \in\left[0, T_{f}\right]$,

$$
\begin{array}{lll}
u_{h}(t) \rightarrow u(t), & \text { weakly in } & H^{1}\left(\Omega, \mathbb{R}^{2}\right), \\
e_{h}(t) \rightarrow e(t), & \text { weakly in } & L^{2}\left(\Omega, \mathbb{M}_{\text {sym }}^{2 \times 2}\right), \\
p_{h}(t) \rightarrow p(t), & \text { weakly in } & L^{2}\left(\Omega, \mathbb{M}_{\text {sym }}^{2 \times 2}\right), \\
v_{h}(t) \rightarrow v(t), & \text { weakly in } & H^{1}(\Omega, \mathbb{R}),
\end{array}
$$

and for all $t \in\left[0, T_{f}\right]$,

$$
(u(t), e(t), p(t)) \in A_{a d m} .
$$


Thanks to the previous convergences, we also have that for a.e. $t \in\left[0, T_{f}\right]$,

$$
(\dot{u}(t), \dot{e}(t), \dot{p}(t)) \in A_{\text {adm }} .
$$

Since $v_{h}$ is uniformly bounded in $W^{1, \infty}\left(0, T_{f}, H^{1}(\Omega, \mathbb{R})\right)$, the Arzelà-Ascoli Theorem for metric spaces [10] implies that $v_{h} \longrightarrow v$ strongly in $C\left(0, T_{f}, L^{2}(\Omega, \mathbb{R})\right)$. Since $0 \leq v_{h}(t) \leq 1$ a.e. in $\Omega$, for all $t$, this convergence implies $a_{h} \longrightarrow a$ in $C\left(0, T_{f}, L^{2}(\Omega, \mathbb{R})\right)$. On the other hand, since for all $t \in\left(0, T_{f}\right]$,

$$
\begin{aligned}
& \left\|a_{h}(t)-a_{h}^{-}(t)\right\|_{L^{2}} \leq 2 h\left\|\dot{a_{h}}(t)\right\|_{L^{2}}, \\
& \left\|e_{h}(t)-e_{h}^{+}(t)\right\|_{L^{2}} \leq h\left\|\dot{e}_{h}(t)\right\|_{L^{2}}, \\
& \left\|v_{h}(t)-v_{h}^{+}(t)\right\|_{L^{2}} \leq h\left\|\dot{v}_{h}(t)\right\|_{L^{2}}
\end{aligned}
$$

we also have that

$$
\begin{array}{clll}
a_{h}^{-} \longrightarrow a & \text { strongly in } & L^{\infty}\left(0, T_{f}, L^{2}(\Omega, \mathbb{R})\right) \text { with } a=v^{2}+\eta, \\
e_{h}^{+} \rightarrow e & \text { weakly* in } & L^{\infty}\left(0, T_{f}, L^{2}\left(\Omega, \mathbb{M}_{\mathrm{sym}}^{2 \times 2}\right)\right), \\
v_{h}^{+} \rightarrow v & \text { weakly* in } & L^{\infty}\left(0, T_{f}, H^{1}(\Omega, \mathbb{R})\right) .
\end{array}
$$

From (3.31) and (3.35), we deduce that for all $t \in\left[0, T_{f}\right]$,

$$
e_{h}^{+}(t)-e(t), \quad \text { weakly in } \quad L^{2}\left(\Omega, \mathbb{M}_{\text {sym }}^{2 \times 2}\right) .
$$

\subsection{Passage to the limit in the equilibrium condition}

Theorem 3.10. For a.e. $t \in\left[0, T_{f}\right]$,

$$
\begin{array}{cl}
-\operatorname{div}(\sigma(t))=f(t), & \text { a.e. in } \Omega, \\
\sigma(t) . \boldsymbol{n}=g(t), & \text { a.e. on } \partial \Omega_{N}
\end{array}
$$

where $\sigma(t)=a(t) A e(t)+\beta E \dot{u}(t)$ and $a(t)=[v(t)]^{2}+\eta$.

Proof. With the previous notation, we can rewrite (3.4) as

$$
\int_{\Omega}\left(a_{h}^{-}(t) A e_{h}^{+}(t)+\beta E \dot{u_{h}}(t)\right): E \phi \mathrm{d} x=\left\langle l_{h}^{+}(t), \phi\right\rangle, \forall \phi \in H_{D}^{1} \forall t \in\left(0, T_{f}\right) .
$$

Integrating (3.40) on fixed $\left[t_{1}, t_{2}\right] \subset\left[0, T_{f}\right]$ we have

$$
\int_{t_{1}}^{t_{2}} \int_{\Omega}\left(a_{h}^{-}(t) A e_{h}^{+}(t)+\beta E \dot{u}_{h}(t)\right): E \phi \mathrm{d} x \mathrm{~d} t=\int_{t_{1}}^{t_{2}}\left\langle l_{h}^{+}(t), \phi\right\rangle \mathrm{d} t .
$$

We now pass to the limit when $h$ goes to zero in (3.41). For any fixed $t_{1}, t_{2} \in\left[0, T_{f}\right], \forall \phi \in H_{D}^{1}$, we write (3.41) as:

$$
\begin{aligned}
0= & \int_{t_{1}}^{t_{2}} \int_{\Omega}\left(a_{h}^{-}(t) A e_{h}^{+}(t)+\beta E \dot{u}_{h}(t)\right): E \phi \mathrm{d} x \mathrm{~d} t-\int_{t_{1}}^{t_{2}}\left\langle l_{h}^{+}(t), \phi\right\rangle \mathrm{d} t \\
= & \int_{t_{1}}^{t_{2}} \int_{\Omega}\left(a(t) A e_{h}^{+}(t)+\beta E \dot{u}_{h}(t)\right): E \phi \mathrm{d} x-\langle l(t), \phi\rangle \mathrm{d} t \\
& +\int_{t_{1}}^{t_{2}}\left\{\int_{\Omega}\left(\left(a_{h}^{-}(t)-a(t)\right) A e_{h}^{+}(t): E \phi \mathrm{d} x\right\} \mathrm{d} t\right. \\
& -\int_{t_{1}}^{t_{2}}\left\langle l_{h}^{+}(t)-l(t), \phi\right\rangle \mathrm{d} t .
\end{aligned}
$$


We estimate the second term on the right-hand side of (3.42) thanks to the Cauchy-Schwarz inequality

$$
\begin{aligned}
& \int_{t_{1}}^{t_{2}} \int_{\Omega}\left(a_{h}^{-}(t)-a(t)\right) A e_{h}^{+}(t): E \phi \mathrm{d} x \mathrm{~d} t \\
& \leq\left\|\left(a_{h}^{-}-a\right) E \phi\right\|_{L^{2}\left(0, T_{f}, L^{2}\right)}\left\|A e_{h}^{+}\right\|_{L^{2}\left(0, T_{f}, L^{2}\right)} .
\end{aligned}
$$

Since $\left(a_{h}^{-}-a\right) \rightarrow 0$ a.e. in $\Omega \times\left(0, T_{f}\right),\left|a_{h}^{-}-a\right|^{2} \leq 4,|\nabla \phi|^{2} \in L^{1}\left(\Omega \times\left(0, T_{f}\right)\right)$ and using Lemma 3.7 we obtain by the Lebesgue dominated convergence

$$
\lim _{h \searrow 0} \int_{t_{1}}^{t_{2}} \int_{\Omega}\left(a_{h}^{-}(t)-a(t)\right) A e_{h}^{+}(t): E \phi \mathrm{d} x \mathrm{~d} t=0
$$

We estimate the last term on the right-hand side of (3.42) by

$$
\int_{t_{1}}^{t_{2}}\left\langle l_{h}^{+}(t)-l(t), \phi\right\rangle \mathrm{d} t \leq h\left(t_{2}-t_{1}\right)\|i\|_{C\left(0, T_{f},\left(H_{D}^{1}\right)^{*}\right)}\|\phi\|_{H^{1}}
$$

Using (3.30), (3.37), (3.42), (3.44), (3.45) we deduce that $\forall\left[t_{1}, t_{2}\right] \subset\left[0, T_{f}\right]$,

$$
\int_{t_{1}}^{t_{2}} \int_{\Omega}(a(t) A e(t)+\beta E \dot{u}(t)): E \phi \mathrm{d} x-\langle l(t), \phi\rangle \mathrm{d} t=0 .
$$

\subsection{Strong compactness result for the elastic strain}

To pass to the limit in the discrete plastic flow rule and crack propagation condition, we need the strong compactness result for the elastic strain contained in the following lemma.

Lemma 3.11. Suppose that for all $t \in\left[0, T_{f}\right), a_{h}^{-}(t) \rightarrow a(t)$ in $L^{2}(\Omega)$ and $e_{h}^{+}(t) \rightarrow e(t)$ weakly in $L^{2}\left(\Omega, \mathbb{M}_{\text {sym }}^{2 \times 2}\right)$. Then for all $t \in\left[0, T_{f}\right), a_{h}^{-}(t) A e_{h}^{+}(t) \rightarrow a(t) A e(t)$ weakly in $L^{2}\left(\Omega, \mathbb{M}_{\text {sym }}^{2 \times 2}\right)$.

Proof. Let $\phi \in L^{2}\left(\Omega, \mathbb{M}_{\text {sym }}^{2 \times 2}\right)$ a test function. We can write

$$
\begin{aligned}
\int_{\Omega}\left(a_{h}^{-}(t) A e_{h}^{+}(t)-a(t) A e(t)\right): \phi \mathrm{d} x= & \int_{\Omega}\left(a_{h}^{-}(t)-a(t)\right) A e_{h}^{+}(t): \phi \mathrm{d} x \\
& +\int_{\Omega} a(t)\left(A e_{h}^{+}(t)-A e(t)\right): \phi \mathrm{d} x .
\end{aligned}
$$

From Lemma 3.7 we deduce that for all $t \in\left[0, T_{f}\right),\left\|A e_{h}^{+}(t)\right\|_{L^{\infty}} \leq C$, so that since $a_{h}^{-}(t) \rightarrow a(t)$ in $L^{2}(\Omega)$ the first term on the right-hand side converges to zero using the Cauchy-Schwarz inequality. Since $e_{h}^{+}(t) \rightarrow e(t)$ in $L^{2}\left(\Omega, \mathbb{M}_{\text {sym }}^{2 \times 2}\right)$, the second term on the right-hand converges to zero.

Proposition 3.12. The following strong convergences hold:

$$
e_{h}, e_{h}^{+} \rightarrow e \quad \text { strongly in } \quad L^{2}\left(0, T_{f}, L^{2}\left(\Omega, \mathbb{M}_{\text {sym }}^{2 \times 2}\right)\right) .
$$


Proof. Given $n \in\left\{1, \ldots, N_{f}\right\}$, we define $[t]_{h}:=t_{h}^{n}$ if $t \in\left(t_{h}^{n-1}, t_{h}^{n}\right]$. We set $t_{1}=0, t_{2}=[t]_{h}$ and $\phi=\dot{u}_{h}$ in $(3.46)$ and (3.41), and substract these two relations:

$$
\begin{aligned}
& \int_{0}^{[t]_{h}} \int_{\Omega}\left(\left(a_{h}^{-}(s) A e_{h}^{+}(s)+\beta E \dot{u_{h}}(s)\right)-(a(s) A e(s)+\beta E \dot{u}(s))\right): E \dot{u_{h}}(s) \mathrm{d} x \mathrm{~d} s \\
= & \int_{0}^{[t]_{h}}\left\langle l_{h}^{+}(s)-l(s), \dot{u_{h}}(s)\right\rangle \mathrm{d} s .
\end{aligned}
$$

We define

$$
f_{h}(t):=\int_{0}^{[t]_{h}} \int_{\Omega}\left(\left(a_{h}^{-}(s) A e_{h}^{+}(s)+\beta E \dot{u_{h}}(s)\right)-(a(s) A e(s)+\beta E \dot{u}(s))\right): E \dot{u_{h}}(s) \mathrm{d} x \mathrm{~d} s .
$$

Using inequality (3.45) with $\phi=\dot{u_{h}}$ and thanks to Proposition 3.6, we have

$$
\lim _{h \searrow 0} \int_{0}^{[t]_{h}}\left\langle l_{h}^{+}(s)-l(s), \dot{u_{h}}(s)\right\rangle \mathrm{d} s=0,
$$

and from (3.48) we deduce

$$
\lim _{h \searrow 0} f_{h}(t)=0
$$

We now estimate

$$
\left|f_{h}(t)\right| \leq\left\|\left(a_{h}^{-} A e_{h}^{+}+\beta E \dot{u_{h}}\right)-(a A e+\beta E \dot{u})\right\|_{L^{2}\left(0, T_{f}, L^{2}\right)}\left\|E \dot{u}_{h}\right\|_{L^{2}\left(0, T_{f}, L^{2}\right)} .
$$

From Proposition 3.6 we deduce that there exists some $C>0$ independent of $h$ such that $\left|f_{h}(t)\right| \leq C$. Thanks to the Lebesgue dominated convergence

$$
\lim _{h \searrow 0} \int_{0}^{T_{f}} f_{h}(t) \mathrm{d} t=0 .
$$

We rewrite $f_{h}(t)$ as

$$
\begin{aligned}
f_{h}(t)= & \int_{0}^{[t]_{h}} \int_{\Omega}\left(\left(a_{h}^{-}(s) A e_{h}^{+}(s)-a(s) A e(s)\right): E \dot{u}_{h}(s) \mathrm{d} x \mathrm{~d} s\right. \\
& +\beta \int_{0}^{[t]_{h}} \int_{\Omega}\left|E \dot{u_{h}}(s)-E \dot{u}(s)\right|^{2} \mathrm{~d} x \mathrm{~d} s \\
& +\beta \int_{0}^{[t]_{h}} \int_{\Omega}\left(E \dot{u_{h}}(s)-E \dot{u}(s)\right): E \dot{u}(s) \mathrm{d} x \mathrm{~d} s .
\end{aligned}
$$

We define

$$
g_{h}(t)=\beta \int_{0}^{[t]_{h}} \int_{\Omega}\left(E \dot{u}_{h}(s)-E \dot{u}(s)\right): E \dot{u}(s) \mathrm{d} x \mathrm{~d} s,
$$

which tends to 0 since $E \dot{u}_{h} \rightarrow E \dot{u}$ weakly in $L^{2}\left(0, T_{f}, L^{2}\left(\Omega, \mathbb{M}_{\text {sym }}^{2 \times 2}\right)\right)$. Using Proposition 3.6 and the Lebesgue dominated convergence theorem yields

$$
\lim _{h \searrow 0} \int_{0}^{T_{f}} g_{h}(t) \mathrm{d} t=0
$$


From (3.49), (3.50), (3.52) we deduce that

$$
\begin{aligned}
& \lim _{h \searrow 0}\left\{\int_{0}^{T_{f}} \int_{0}^{[t]_{h}} \int_{\Omega}\left(a_{h}^{-}(s) A e_{h}^{+}(s)-a(s) A e(s)\right): E \dot{u_{h}}(s) \mathrm{d} x \mathrm{~d} s \mathrm{~d} t\right. \\
+ & \left.\beta \int_{0}^{T_{f}} \int_{0}^{[t]_{h}} \int_{\Omega}\left|E \dot{u}_{h}(s)-E \dot{u}(s)\right|^{2} \mathrm{~d} x \mathrm{~d} s \mathrm{~d} t\right\}=0,
\end{aligned}
$$

so that

$$
\limsup _{h \searrow 0} \int_{0}^{T_{f}} \int_{0}^{[t]_{h}} \int_{\Omega}\left(a_{h}^{-}(s) A e_{h}^{+}(s)-a(s) A e(s)\right): E u_{h}(s) \mathrm{d} x \mathrm{~d} s \mathrm{~d} t \leq 0 .
$$

We now estimate the integral in (3.54). We note that

$$
\begin{aligned}
I_{h}:= & \int_{0}^{T_{f}} \int_{0}^{[t]_{h}} \int_{\Omega}\left(a_{h}^{-}(s) A e_{h}^{+}(s)-a(s) A e(s)\right): E \dot{u_{h}}(s) \mathrm{d} x \mathrm{~d} s \mathrm{~d} t \\
= & \int_{0}^{T_{f}} \int_{0}^{[t]_{h}} \int_{\Omega}\left(a_{h}^{-}(s) A e_{h}^{+}(s)-a(s) A e(s)\right):\left(E \dot{u_{h}}(s)-E \dot{u}(s)\right) \mathrm{d} x \mathrm{~d} s \mathrm{~d} t \\
& +\int_{0}^{T_{f}} \int_{0}^{[t]_{h}} \int_{\Omega}\left(a_{h}^{-}(s) A e_{h}^{+}(s)-a(s) A e(s)\right): E \dot{u}(s) \mathrm{d} x \mathrm{~d} s \mathrm{~d} t \\
= & \int_{0}^{T_{f}} \int_{0}^{[t]_{h}} \int_{\Omega} a_{h}^{-}(s) A e_{h}^{+}(s):\left(E u_{h}(s)-E \dot{u}(s)\right) \mathrm{d} x \mathrm{~d} s \mathrm{~d} t \\
& -\int_{0}^{T_{f}} \int_{0}^{[t]_{h}} \int_{\Omega} a(s) A e(s):\left(E \dot{u}_{h}(s)-E \dot{u}(s)\right) \mathrm{d} x \mathrm{~d} s \mathrm{~d} t \\
& +\int_{0}^{T_{f}} \int_{0}^{[t]_{h}} \int_{\Omega}\left(a_{h}^{-}(s)-a(s)\right) A e_{h}^{+}(s): E \dot{u}(s) \mathrm{d} x \mathrm{~d} s \mathrm{~d} t \\
& +\int_{0}^{T_{f}} \int_{0}^{[t]_{h}} \int_{\Omega} a(s)\left(A e_{h}^{+}(s)-A e(s)\right): E \dot{u}(s) \mathrm{d} x \mathrm{~d} s \mathrm{~d} t \\
= & I_{h}^{1}+I_{h}^{2}+I_{h}^{3}+I_{h}^{4} .
\end{aligned}
$$

Since $E \dot{u}_{h} \rightarrow E \dot{u}$ weakly in $L^{2}\left(0, T_{f}, L^{2}\left(\Omega, \mathbb{M}_{\mathrm{sym}}^{2 \times 2}\right)\right)$, and $e_{h}^{+} \rightarrow e$ weakly in $L^{2}\left(0, T_{f}, L^{2}\left(\Omega, \mathbb{M}_{\mathrm{sym}}^{2 \times 2}\right)\right)$, the Lebesque's dominated convergence shows that

$$
\begin{aligned}
I_{h}^{2} & :=-\int_{0}^{T_{f}} \int_{0}^{[t]_{h}} \int_{\Omega} a(s) A e(s):\left(E \dot{u_{h}}(s)-E \dot{u}(s)\right) \mathrm{d} x \mathrm{~d} s \mathrm{~d} t \rightarrow 0 \\
I_{h}^{4} & :=\int_{0}^{T_{f}} \int_{0}^{[t]_{h}} \int_{\Omega} a(s)\left(A e_{h}^{+}(s)-A e(s)\right): E \dot{u}(s) \mathrm{d} x \mathrm{~d} s \mathrm{~d} t \rightarrow 0 .
\end{aligned}
$$

Since $a_{h}^{-}-a \rightarrow 0$ a.e. in $\Omega \times\left(0, T_{f}\right)$ and $a \in L^{\infty}\left(\left(0, T_{f}\right) \times \Omega\right)$, and using Lemma 3.7, Proposition 3.6, and Lebesgue's dominated convergence theorem imply

$$
I_{h}^{3}:=\int_{0}^{T_{f}} \int_{0}^{[t]_{h}} \int_{\Omega}\left(a_{h}^{-}(s)-a(s)\right) A e_{h}^{+}(s): E \dot{u}(s) \mathrm{d} x \mathrm{~d} s \mathrm{~d} t \rightarrow 0 .
$$


We deduce that

$$
\limsup _{h \searrow 0} I_{h}=\limsup _{h \searrow 0} I_{h}^{1} \leq 0
$$

By the kinematic compatibility, $E \dot{u_{h}}-E \dot{u}=\left(\dot{e_{h}}-\dot{e}\right)+\left(\dot{p_{h}}-\dot{p}\right)$ we have

$$
\begin{aligned}
\limsup _{h \searrow 0} I_{h}^{1}= & \limsup _{h \searrow 0}\left\{\int_{0}^{T_{f}} \int_{0}^{[t]_{h}} \int_{\Omega} a_{h}^{-}(s) A e_{h}^{+}(s):\left(\dot{e_{h}}(s)-\dot{e}(s)\right) \mathrm{d} x \mathrm{~d} s \mathrm{~d} t\right. \\
& \left.+\int_{0}^{T_{f}} \int_{0}^{[t]_{h}} \int_{\Omega} a_{h}^{-}(s) A e_{h}^{+}(s):\left(\dot{p_{h}}(s)-\dot{p}(s)\right) \mathrm{d} x \mathrm{~d} s \mathrm{~d} t\right\} . \\
: & =\limsup _{h \searrow 0}\left\{K_{h}^{1}+K_{h}^{2}\right\} .
\end{aligned}
$$

We can write

$$
\begin{aligned}
K_{h}^{1}:= & \int_{0}^{T_{f}} \int_{0}^{[t]_{h}} \int_{\Omega} a_{h}^{-}(s) A e_{h}^{+}(s):\left(\dot{e_{h}}(s)-\dot{e}(s)\right) \mathrm{d} x \mathrm{~d} s \mathrm{~d} t \\
= & -\int_{0}^{T_{f}} \int_{0}^{[t]_{h}} \int_{\Omega}\left(a_{h}(s)-a_{h}^{-}(s)\right) A e_{h}^{+}(s):\left(\dot{e_{h}}(s)-\dot{e}(s)\right) \mathrm{d} x \mathrm{~d} s \mathrm{~d} t \\
& +\int_{0}^{T_{f}} \int_{0}^{[t]_{h}} \int_{\Omega} a_{h}(s) A e_{h}^{+}(s):\left(\dot{e_{h}}(s)-\dot{e}(s)\right) \mathrm{d} x \mathrm{~d} s \mathrm{~d} t .
\end{aligned}
$$

From (3.34), we deduce that $\left(a_{h}^{-}-a_{h}\right) \rightarrow 0$ a.e. in $\Omega \times\left(0, T_{f}\right)$. Lemma 3.7, Proposition 3.6 and the Lebesgue's dominated convergence give

$$
\int_{0}^{T_{f}} \int_{0}^{[t]_{h}} \int_{\Omega}\left(a_{h}(s)-a_{h}^{-}(s)\right) A e_{h}^{+}(s):\left(\dot{e_{h}}(s)-\dot{e}(s)\right) \mathrm{d} x \mathrm{~d} s \mathrm{~d} t \rightarrow 0,
$$

so that (3.58) and (3.59) imply

$$
\limsup _{h \searrow 0} K_{h}^{1}=\limsup _{h \searrow 0} \int_{0}^{T_{f}} \int_{0}^{[t]_{h}} \int_{\Omega} a_{h}(s) A e_{h}^{+}(s):\left(\dot{e_{h}}(s)-\dot{e}(s)\right) \mathrm{d} x \mathrm{~d} s \mathrm{~d} t .
$$

Furthermore,

$$
\begin{aligned}
J_{h}^{1}:= & \int_{0}^{T_{f}} \int_{0}^{[t]_{h}} \int_{\Omega} a_{h}(s) A e_{h}^{+}(s):\left(\dot{e_{h}}(s)-\dot{e}(s)\right) \mathrm{d} x \mathrm{~d} s \mathrm{~d} t \\
= & \int_{0}^{T_{f}} \int_{0}^{[t]_{h}} \int_{\Omega} a_{h}(s)\left(A e_{h}^{+}(s)-A e_{h}(s)\right):\left(\dot{e_{h}}(s)-\dot{e}(s)\right) \mathrm{d} x \mathrm{~d} s \mathrm{~d} t \\
& +\int_{0}^{T_{f}} \int_{0}^{[t]_{h}} \int_{\Omega} a_{h}(s) A\left(e_{h}(s)-e(s)\right):\left(\dot{e_{h}}(s)-\dot{e}(s)\right) \mathrm{d} x \mathrm{~d} s \mathrm{~d} t \\
& +\int_{0}^{T_{f}} \int_{0}^{[t]_{h}} \int_{\Omega}\left(a_{h}(s)-a(s)\right) A e(s):\left(\dot{e_{h}}(s)-\dot{e}(s)\right) \mathrm{d} x \mathrm{~d} s \mathrm{~d} t \\
& +\int_{0}^{T_{f}} \int_{0}^{[t]_{h}} \int_{\Omega} a(s) A e(s):\left(\dot{e_{h}}(s)-\dot{e}(s)\right) \mathrm{d} x \mathrm{~d} s \mathrm{~d} t \\
= & L_{h}^{5}+L_{h}^{6}+L_{h}^{7}+L_{h}^{8} .
\end{aligned}
$$


The strong convergence $e_{h}-e_{h}^{+} \rightarrow 0$ in $L^{2}\left(0, T_{f}, L^{2}\left(\Omega, \mathbb{M}_{\text {sym }}^{2 \times 2}\right)\right.$ ) (see (3.35)), the weak convergence $\dot{e_{h}} \rightarrow \dot{e}$ in $L^{2}\left(0, T_{f}, L^{2}\left(\Omega, \mathbb{M}_{\text {sym }}^{2 \times 2}\right)\right)$, Propostion 3.6 and Lebesgue convergence dominated theorem yield to

$$
\begin{gathered}
L_{h}^{5}:=\int_{0}^{T_{f}} \int_{0}^{[t]_{h}} \int_{\Omega} a_{h}(s)\left(A e_{h}^{+}(s)-A e_{h}(s)\right):\left(\dot{e_{h}}(s)-\dot{e}(s)\right) \mathrm{d} x \mathrm{~d} s \mathrm{~d} t \rightarrow 0, \\
L_{h}^{8}:=\int_{0}^{T_{f}} \int_{0}^{[t]_{h}} \int_{\Omega} a(s) A e(s):\left(\dot{e_{h}}(s)-\dot{e}(s)\right) \mathrm{d} x \mathrm{~d} s \mathrm{~d} t \rightarrow 0 .
\end{gathered}
$$

We now estimate

$$
L_{h}^{7}:=\int_{0}^{T_{f}} \int_{0}^{[t]_{h}} \int_{\Omega}\left(a_{h}(s)-a(s)\right) A e(s):\left(\dot{e_{h}}(s)-\dot{e}(s)\right) \mathrm{d} x \mathrm{~d} s \mathrm{~d} t .
$$

We have

$$
\begin{aligned}
& \int_{0}^{[t]_{h}} \int_{\Omega}\left(a_{h}(s)-a(s)\right) A e(s):\left(\dot{e_{h}}(s)-\dot{e}(s)\right) \mathrm{d} x \mathrm{~d} s \\
& \leq\left\|\left(a_{h}-a\right) A e\right\|_{L^{2}\left(0, T_{f}, L^{2}\right)}\left\|\dot{e_{h}}-\dot{e}\right\|_{L^{2}\left(0, T_{f}, L^{2}\right)} .
\end{aligned}
$$

From Proposition 3.6 we deduce that there exists some $C>0$ independent of $h$ such that

$$
\int_{0}^{[t]_{h}} \int_{\Omega}\left(a_{h}(s)-a(s)\right) A e(s):\left(\dot{e_{h}}(s)-\dot{e}(s)\right) \mathrm{d} x \mathrm{~d} s \leq C\left\|\left(a_{h}-a\right) A e\right\|_{L^{2}\left(0, T_{f}, L^{2}\right)} .
$$

Since $a_{h}(t) \in L^{\infty}(\Omega),\left(a_{h}-a\right) \rightarrow 0$ a.e. in $\Omega \times\left(0, T_{f}\right)$, the Lebesgue's dominated convergence gives that

$$
L_{h}^{7} \rightarrow 0
$$

From (3.60), (3.61), (3.62), (3.63), (3.64) we deduce that

$$
\limsup _{h \backslash 0} K_{h}^{1}=\limsup _{h \backslash 0} \int_{0}^{T_{f}} \int_{0}^{[t]_{h}} \int_{\Omega} a_{h}(s) A\left(e_{h}(s)-e(s)\right)\left(\dot{e_{h}}(s)-\dot{e}(s)\right) \mathrm{d} x \mathrm{~d} s \mathrm{~d} t .
$$

We now estimate $K_{h}^{2}$ :

$$
\begin{aligned}
K_{h}^{2} & :=\int_{0}^{T_{f}} \int_{0}^{[t]_{h}} \int_{\Omega} a_{h}^{-}(s) A e_{h}^{+}(s):\left(\dot{p_{h}}(s)-\dot{p}(s)\right) \mathrm{d} x \mathrm{~d} s \mathrm{~d} t \\
& =\int_{0}^{T_{f}} \int_{0}^{[t]_{h}} \int_{\Omega}\left(a_{h}^{-}(s) A e_{h}^{+}(s): \dot{p_{h}}(s)\right)-\left(a_{h}^{-}(s) A e_{h}^{+}(s): \dot{p}(s)\right) \mathrm{d} x \mathrm{~d} s \mathrm{~d} t .
\end{aligned}
$$

Using Lemma 3.11, we have $a_{h}^{-} A e_{h}^{+}(t) \rightarrow a A e(t)$ weakly in $L^{2}\left(\Omega, \mathbb{M}_{\text {sym }}^{2 \times 2}\right)$. Thanks to Lemma 3.7, we also have that for all $t \in\left[0, T_{f}\right), a_{h}^{-}(t) A e_{h}^{+}(t) \in K$ a.e. in $\Omega$, with $\mathrm{K}$ a convex closed set. We obtain that for all $t \in\left[0, T_{f}\right)$, $a(t) A e(t) \in K$ a.e. in $\Omega$. By Lemma 3.8, we have for all $t \in\left(0, T_{f}\right), a_{h}^{-}(t) A e_{h}^{+}(t): \dot{p_{h}}(t) \geqslant a(t) A e(t): \dot{p_{h}}(t)$ a.e. in $\Omega$. So that we have

$$
\begin{aligned}
K_{h}^{2} \geqslant & \int_{0}^{T_{f}} \int_{0}^{[t]_{h}} \int_{\Omega} a(s) A e(s): \dot{p_{h}}(s)-a_{h}^{-}(s) A e_{h}^{+}(s): \dot{p}(s) \mathrm{d} x \mathrm{~d} s \mathrm{~d} t \\
= & \int_{0}^{T_{f}} \int_{0}^{[t]_{h}} \int_{\Omega} a(s) A e(s):\left(\dot{p_{h}}(s)-\dot{p}(s)\right) \mathrm{d} x \mathrm{~d} s \mathrm{~d} t \\
& +\int_{0}^{T_{f}} \int_{0}^{[t]_{h}} \int_{\Omega}\left(a(s) A e(s)-a_{h}^{-}(s) A e_{h}^{+}(s)\right): \dot{p}(s) \mathrm{d} x \mathrm{~d} s \mathrm{~d} t .
\end{aligned}
$$


Since $\dot{p_{h}} \rightarrow \dot{p}$ weakly in $L^{2}\left(0, T_{f}, L^{2}\left(\Omega, \mathbb{M}_{\text {sym }}^{2 \times 2}\right)\right)$,

$$
\int_{0}^{T_{f}} \int_{0}^{[t]_{h}} \int_{\Omega} a(s) A e(s):\left(\dot{p_{h}}(s)-\dot{p}(s)\right) \mathrm{d} x \mathrm{~d} s \mathrm{~d} t \rightarrow 0 .
$$

Since $a_{h}^{-} A e_{h}^{+} \rightarrow a A e$ weakly in $L^{2}\left(0, T_{f}, L^{2}\left(\Omega, \mathbb{M}_{\mathrm{sym}}^{2 \times 2}\right)\right)$

$$
\int_{0}^{T_{f}} \int_{0}^{[t]_{h}} \int_{\Omega}\left(a(s) A(s)-a_{h}^{-}(s)\right) A e_{h}^{+}(s): \dot{p}(s) \mathrm{d} x \mathrm{~d} s \mathrm{~d} t \rightarrow 0 .
$$

From (3.56), (3.57), (3.65), (3.67), (3.68), (3.69) we deduce that

$$
\limsup _{h \searrow 0} I_{h} \geqslant \limsup _{h \searrow 0} \int_{0}^{T_{f}} \int_{0}^{[t]_{h}} \int_{\Omega} a_{h}(s) A\left(e_{h}(s)-e(s)\right):\left(\dot{e_{h}}(s)-\dot{e}(s)\right) \mathrm{d} x \mathrm{~d} s \mathrm{~d} t .
$$

Integrating by parts and using the fact that $e_{h}(0)=e_{0}$, we get

$$
\begin{aligned}
& \int_{0}^{[t]_{h}} \int_{\Omega} a_{h}(s) A\left(e_{h}(s)-e(s)\right):\left(\dot{e_{h}}(s)-\dot{e}(s)\right) \mathrm{d} x \mathrm{~d} s \\
= & \int_{\Omega} \frac{1}{2} a_{h}\left([t]_{h}\right) A\left(e_{h}\left([t]_{h}\right)-e\left([t]_{h}\right)\right):\left(e_{h}\left([t]_{h}\right)-e\left([t]_{h}\right)\right) \mathrm{d} x \\
& -\frac{1}{2} \int_{0}^{[t]_{h}} \int_{\Omega} \dot{a}_{h}(s) A\left(e_{h}(s)-e(s)\right):\left(e_{h}(s)-e(s)\right) \mathrm{d} x \mathrm{~d} s .
\end{aligned}
$$

Since $\forall t \in\left(0, T_{f}\right]$ and a.e. in $\Omega, \dot{a}_{h}(., t) \leq 0$,

$$
-\int_{0}^{T_{f}} \int_{0}^{[t]_{h}} \int_{\Omega} \dot{a_{h}}(s) A\left(e_{h}(s)-e(s)\right):\left(e_{h}(s)-e(s)\right) \mathrm{d} x \mathrm{~d} s \mathrm{~d} t \geqslant 0 .
$$

Since $a_{h}(t) \geqslant \eta$ for all $t \in\left[0, T_{f}\right]$, using the ellipticity hypothesis $A e: e \geqslant \alpha_{A}|e|^{2}$, we deduce from (3.70), and (3.71) that

$$
\limsup _{h \searrow 0} I_{h} \geqslant \frac{1}{2} \eta \alpha_{1} \limsup _{h \searrow 0} \int_{0}^{T_{f}} \int_{\Omega}\left|e_{h}\left([t]_{h}\right)-e\left([t]_{h}\right)\right|^{2} \mathrm{~d} x \mathrm{~d} t .
$$

By definition we have $e_{h}\left([t]_{h}\right)=e_{h}^{+}(t)$. Since $e \in W^{1, \infty}\left(0, T_{f}, L^{2}\left(\Omega, \mathbb{M}_{\mathrm{sym}}^{2 \times 2}\right)\right)$, we now estimate $e(t)-e\left([t]_{h}\right)$ in $L^{2}$ using Cauchy-Schwarz inequality and Fubini theorem:

$$
\begin{aligned}
& \int_{\Omega}\left|e\left([t]_{h}\right)-e(t)\right|^{2} \mathrm{~d} x \leq \int_{\Omega}\left|\int_{t}^{[t]_{h}} \dot{e}(s) \mathrm{d} s\right|^{2} \mathrm{~d} x \\
\leq & h^{1 / 2} \int_{\Omega} \int_{t}^{[t]_{h}}|\dot{e}(s)|^{2} \mathrm{~d} s \mathrm{~d} x \leq C h^{1 / 2}\|\dot{e}\|_{L^{\infty}\left(0, T_{f}, L^{2}\right)} .
\end{aligned}
$$

We define for $t \in\left(0, T_{f}\right], e^{h}(t):=e\left([t]_{h}\right)$. From (3.73) we deduce that $e^{h} \rightarrow e$ strongly in $L^{\infty}\left(0, T_{f}, L^{2}\left(\Omega, \mathbb{M}_{\mathrm{sym}}^{2 \times 2}\right)\right)$. Since $e_{h}\left([t]_{h}\right)-e\left([t]_{h}\right)=e_{h}^{+}(t)-e\left([t]_{h}\right)=e_{h}^{+}(t)-e(t)+e(t)-e\left([t]_{h}\right)$, we deduce from (3.54) and (3.72) that

$$
\begin{aligned}
0 \geqslant & \limsup _{h \searrow 0} I_{h} \geqslant \frac{\eta \alpha_{1}}{2} \limsup _{h \searrow 0}\left\{\int_{0}^{T_{f}}\left\|e_{h}^{+}(t)-e(t)\right\|_{L^{2}}^{2} \mathrm{~d} t\right. \\
& \left.+2 \int_{0}^{T_{f}} \int_{\Omega}\left(e_{h}^{+}(t)-e(t)\right):\left(e(t)-e\left([t]_{h}\right)\right) \mathrm{d} x \mathrm{~d} t+\int_{0}^{T_{f}}\left\|e\left([t]_{h}\right)-e(t)\right\|_{L^{2}}^{2} \mathrm{~d} t\right\} .
\end{aligned}
$$


Since $e^{h} \rightarrow e$ strongly in $L^{\infty}\left(0, T_{f}, L^{2}\left(\Omega, \mathbb{M}_{\mathrm{sym}}^{2 \times 2}\right)\right)$ and $e_{h}^{+} \rightarrow e$ weakly* in $L^{\infty}\left(0, T_{f}, L^{2}\left(\Omega, \mathbb{M}_{\mathrm{sym}}^{2 \times 2}\right)\right)$, it follows that

$$
\begin{gathered}
\int_{0}^{T_{f}} \int_{\Omega}\left(e_{h}^{+}(t)-e(t)\right):\left(e(t)-e\left([t]_{h}\right)\right) \mathrm{d} x \mathrm{~d} t \rightarrow 0, \\
\int_{0}^{T_{f}}\left\|e\left([t]_{h}\right)-e(t)\right\|_{L^{2}}^{2} \mathrm{~d} t \rightarrow 0 .
\end{gathered}
$$

We deduce from (3.74), (3.75), (3.76) that

$$
0 \geqslant \limsup _{h \searrow 0} \int_{0}^{T_{f}}\left\|e_{h}^{+}(t)-e(t)\right\|_{L^{2}}^{2} \mathrm{~d} t \geqslant \liminf _{h \searrow 0} \int_{0}^{T_{f}}\left\|e_{h}^{+}(t)-e(t)\right\|_{L^{2}}^{2} \mathrm{~d} t \geqslant 0 .
$$

We conclude that

$$
\lim _{h \searrow 0} \int_{0}^{T_{f}}\left\|e_{h}^{+}(t)-e(t)\right\|_{L^{2}}^{2} \mathrm{~d} t=0
$$

and as a consequence of (3.35) we deduce

$$
\lim _{h \searrow 0} \int_{0}^{T_{f}}\left\|e_{h}(t)-e(t)\right\|_{L^{2}}^{2} \mathrm{~d} t=0 .
$$

We now derive the plastic flow rule and the minimality of $v$.

\subsection{Passage to the limit in the plastic flow rule}

Corollary 3.13. For a.e. $t \in\left[0, T_{f}\right]$,

$$
a(t) A e(t) \in \partial H(\dot{p}(t)) \quad \text { a.e.in } \Omega .
$$

Proof. For all $q \in L^{2}\left(\left[0, T_{f}\right], L^{2}(\Omega, K)\right)$, we have that

$$
\int_{0}^{T_{f}} \int_{\Omega}\left(a_{h}^{-}(t) A e_{h}^{+}(t)-q\right): \dot{p}_{h}(t) \mathrm{d} x \mathrm{~d} t \geqslant 0 .
$$

We can rewrite

$$
\begin{aligned}
& \int_{0}^{T_{f}} \int_{\Omega}\left(a_{h}^{-}(t) A e_{h}^{+}(t)-q\right): \dot{p}_{h}(t) \mathrm{d} x \mathrm{~d} t \\
= & \int_{0}^{T_{f}} \int_{\Omega}\left(a_{h}^{-}(t)-a(t)\right) A e_{h}^{+}(t): \dot{p}_{h}(t) \mathrm{d} x \mathrm{~d} t \\
& +\int_{0}^{T_{f}} \int_{\Omega} a(t)\left(A e_{h}^{+}(t)-e(t)\right): \dot{p}_{h}(t) \mathrm{d} x \mathrm{~d} t \\
& +\int_{0}^{T_{f}} \int_{\Omega}(a(t) A e(t)-q): \dot{p}_{h}(t) \mathrm{d} x \mathrm{~d} t . \\
= & : T_{1}+T_{2}+T_{3} .
\end{aligned}
$$


By Lebesgue dominated convergence theorem, Proposition 3.6 and Lemma 3.7, $T_{1}$ converges to zero. Thanks to Propostion 3.12, $T_{2}$ converges to zero. Since $\dot{p_{h}} \rightarrow \dot{p}$ weakly in $L^{2}\left(0, T_{f}, L^{2}\right), T_{3} \rightarrow \int_{0}^{T_{f}} \int_{\Omega}(a(t) A e(t)-q)$ : $\dot{p}(t) \mathrm{d} x \mathrm{~d} t$. We obtain

$$
\int_{0}^{T_{f}} \int_{\Omega}(a(t) A e(t)-q): \dot{p}(t) \mathrm{d} x \mathrm{~d} t \geqslant 0 .
$$

Since $a(t) A e(t) \in K$ a.e. in $\Omega$ for all $t \in\left[0, T_{f}\right)$, inequality (3.81) gives that for a.e. $t \in\left[0, T_{f}\right], \dot{p}(t) \in$ $\partial 1_{K}(a(t) A e(t))=\partial H^{*}(a(t) A e(t))$ a.e. in $\Omega$. And we conclude by convex duality.

\subsection{Passage to the limit in the crack propagation condition}

Corollary 3.14. For a.e. $t \in\left[0, T_{f}\right]$,

$$
\begin{aligned}
2 \varepsilon \int_{\Omega} \nabla v(t) \nabla(v(t)-\varphi) \mathrm{d} x & +\int_{\Omega} v(t) A e(t): e(t)(v(t)-\varphi) \mathrm{d} x \\
& +(2 \varepsilon)^{-1} \int_{\Omega}(v(t)-1)(v(t)-\varphi) \mathrm{d} x \leq 0,
\end{aligned}
$$

for any $\varphi \in H^{1}(\Omega), \varphi \leq v(t)$ and $\varphi=1$ on $\partial \Omega_{D}$.

Proof. For all $t \in\left(0, T_{f}\right]$ we rewrite $(3.8)$ as

$$
\begin{aligned}
& 2 \varepsilon \int_{\Omega} \nabla v_{h}^{+}(t) \nabla\left(v_{h}^{+}(t)-\varphi\right) \mathrm{d} x+\int_{\Omega} v_{h}^{+}(t) A e_{h}^{+}(t): e_{h}^{+}(t)\left(v_{h}^{+}(t)-\varphi\right) \mathrm{d} x \\
+ & (2 \varepsilon)^{-1} \int_{\Omega}\left(v_{h}^{+}(t)-1\right)\left(v_{h}^{+}(t)-\varphi\right) \mathrm{d} x \leq 0
\end{aligned}
$$

for any $\varphi \in H^{1}(\Omega), \varphi \leq v_{h}^{-}(t)$ and $\varphi=1$ on $\partial \Omega_{D}$. Since $v_{h}^{+}(t)$ is minimal among all $\varphi \leq v_{h}^{-}(t)$, it is minimal among $\varphi \leq v_{h}^{+}(t)$. Integrating over $\left[0, T_{f}\right]$, we obtain

$$
\begin{aligned}
& 2 \varepsilon \int_{0}^{T_{f}} \int_{\Omega} \nabla v_{h}^{+}(t) \nabla\left(v_{h}^{+}(t)-\varphi\right) \mathrm{d} x \mathrm{~d} t \\
+ & \int_{0}^{T_{f}} \int_{\Omega} v_{h}^{+}(t) A e_{h}^{+}(t): e_{h}^{+}(t)\left(v_{h}^{+}(t)-\varphi\right) \mathrm{d} x \mathrm{~d} t \\
+ & (2 \varepsilon)^{-1} \int_{0}^{T_{f}} \int_{\Omega}\left(v_{h}^{+}(t)-1\right)\left(v_{h}^{+}(t)-\varphi\right) \mathrm{d} x \mathrm{~d} t \leq 0
\end{aligned}
$$

for any $\varphi \in H^{1}(\Omega), \varphi \leq v_{h}^{+}(t)$ and $\varphi=1$ on $\partial \Omega_{D}$. Let $\alpha \in L^{2}\left(0, T_{f}, H^{1}(\Omega)\right)$ with $\alpha(., t) \geqslant 0$ and $\alpha(., t)=0$ on $\partial \Omega_{D}$ for all $t \in\left[0, T_{f}\right]$. Testing (3.84) with admissible test function $\varphi=v_{h}^{+}(t)-\alpha(t)$ we obtain

$$
\begin{aligned}
& 2 \varepsilon \int_{0}^{T_{f}} \int_{\Omega} \nabla v_{h}^{+}(t) \nabla \alpha(t) \mathrm{d} x \mathrm{~d} t+\int_{0}^{T_{f}} \int_{\Omega} v_{h}^{+}(t) A e_{h}^{+}(t): e_{h}^{+}(t) \alpha(t) \mathrm{d} x \mathrm{~d} t \\
+ & (2 \varepsilon)^{-1} \int_{0}^{T_{f}} \int_{\Omega}\left(v_{h}^{+}(t)-1\right) \alpha(t) \mathrm{d} x \mathrm{~d} t \leq 0
\end{aligned}
$$

for any $\alpha \in L^{2}\left(0, T_{f}, H^{1}(\Omega)\right)$ with $\alpha(., t) \geqslant 0$ and $\alpha(., t)=0$ on $\partial \Omega_{D}$ for all $t \in\left[0, T_{f}\right]$. Since $v_{h}^{+} \stackrel{*}{\rightarrow} v$ in $L^{\infty}\left(0, T_{f}, H^{1}\right), e_{h}^{+} \rightarrow e$ strongly in $L^{2}\left(0, T_{f}, L^{2}\left(\Omega, \mathbb{M}_{\text {sym }}^{2 \times 2}\right)\right)$ and $v_{h}^{+} \rightarrow v$ a.e. in $\Omega \times\left(0, T_{f}\right)$ we obtain

$$
\begin{aligned}
& 2 \varepsilon \int_{0}^{T_{f}} \int_{\Omega} \nabla v(t) \nabla \alpha(t) \mathrm{d} x \mathrm{~d} t+\int_{0}^{T_{f}} \int_{\Omega} v(t) A e(t): e(t) \alpha(t) \mathrm{d} x \mathrm{~d} t \\
+ & (2 \varepsilon)^{-1} \int_{0}^{T_{f}} \int_{\Omega}(v(t)-1) \alpha(t) \mathrm{d} x \mathrm{~d} t \leq 0
\end{aligned}
$$


for any $\alpha \in L^{2}\left(0, T_{f}, H^{1}(\Omega)\right)$ with $\alpha(., t) \geqslant 0$ and $\alpha(., t)=0$ on $\partial \Omega_{D}$ for all $t \in\left[0, T_{f}\right]$. We set $\alpha(t)=v(t)-\varphi(t)$ with $\varphi(., t) \leq v(., t), \varphi \in L^{2}\left(0, T_{f}, H^{1}(\Omega)\right)$ and $\varphi(., t)=1$ on $\partial \Omega_{D}$.

\section{Conclusion}

In this paper, we proved an existence result for a visco-elasto-plastic model with fracture. We studied an associated discrete time evolution model. We proved that as discretization time step converges to zero, the discrete time evolution solutions converge to limit that is a solution of continuous time visco-elasto-plastic model with fracture. In other words, the limit evolution satisfies $(\mathrm{H} 1)-(\mathrm{H} 7)$.

Acknowledgements. The author wish to express his gratitude to E. Bonnetier and S. Labbé for the fruitful and enlightening discussions.

\section{REFERENCES}

[1] L. Ambrosio and V.M. Tortorelli, Approximation of functionals depending on jumps by elliptic functionals via $\Gamma$-convergence. Comm. Pure Appl. Math. XLIII (1990) 999-1036.

[2] J.-F. Babadjian and M.G. Mora, Approximation of dynamic and quasi-static evolution problems in elasto-plasticity by cap models. Quart. Appl. Math. 73 (2015) 265-316.

[3] J.-F. Babadjian, G.A. Francfort and M.G. Mora, Quasistatic evolution in non-associative plasticity - the cap model. SIAM J. Math. Anal. 44 (2012) 245-292.

[4] E. Bonnetier, L. Jakabčin, S. Labbé and A. Replumaz, Numerical simulation of a class of models that combine several mechanisms of dissipation: fracture, plasticity, viscous dissipation. J. Comput. Phys. 271 (2014) 397-414.

[5] B. Bourdin, Une formulation variationnelle en mécanique de la rupture, théorie et mise en oeuvre numérique. Th.D. thesis, Université Paris Nord, France (1998).

[6] B. Bourdin, Numerical implementation of the variational formulation of brittle fracture. Interfaces Free Bound 9 (2007) 411-430.

[7] B. Bourdin, G. Francfort and J.J. Marigo, Numerical experiments in revisited brittle fracture. J. Mech. Phys. Solids 48 (2000) $797-826$.

[8] B. Bourdin, G. Francfort and J.J. Marigo, The variational approach to fracture. J. Elasticity 91 (2008) 1-148.

[9] G. Dal Maso and R. Toader, Quasistatic crack growth in elasto-plastic materials: the two-dimensional case. Arch. Ration. Mech. Anal. 196 (2010) 867-906.

[10] N. Dunford and J.T. Schwartz, Linear operators. Part I. Wiley Classics Library. John Wiley and Sons Inc., New York (1988).

[11] L.C. Evans, Partial differential equations. Grad. Stud. Math. AMS, Rhode Island (1998).

[12] G.A. Francfort and J.-J. Marigo, Revisiting brittle fracture as an energy minimization problem. J. Mech. Phys. Solids 46 (1998) 1319-1342.

[13] A. Griffith, The phenomena of rupture and flow in solids. Philos. Trans. R. Soc. Lond. Ser. A 221 (1920) 133-178.

[14] F. Iurlano, A density result for GSBD and its application to the approximation of brittle fracture energies. Calc. Var. Partial Differ. Eqs. 51 (2014) 315-342.

[15] L. Jakabčin, Modélisation, analyse et simulation numérique de solides combinant plasticité, rupture et dissipation visqueuse. Th.D. thesis, Université de Grenoble, France (2014).

[16] C.J. Larsen, C. Ortner and E. Suli, Existence of solutions to a regularized model of dynamic fracture. M3AS 20 (2010) 1021-1048.

[17] G. Peltzer and P. Tapponnier, Formation and evolution of strike-slip faults, rifts, and basins during the India-Asia collision: An experimental approach. J. Geophys. Res. 93 (1988) 15085-15117. 